University of Nebraska - Lincoln

DigitalCommons@University of Nebraska - Lincoln

May 1993

\title{
FAR-ULTRAVIOLET STELLAR PHOTOMETRY: A FIELD IN MONOCEROS
}

\author{
Edward G. Schmidt \\ University of Nebraska-Lincoln, eschmidt1@unl.edu \\ George R. Carruthers \\ E. O. Hulburt Center for Space Research Naval Research Laboratory, Washington. DC
}

Follow this and additional works at: https://digitalcommons.unl.edu/physicsschmidt

Part of the Physics Commons

Schmidt, Edward G. and Carruthers, George R., "FAR-ULTRAVIOLET STELLAR PHOTOMETRY: A FIELD IN MONOCEROS" (1993). Edward Schmidt Publications. 17.

https://digitalcommons.unl.edu/physicsschmidt/17

This Article is brought to you for free and open access by the Research Papers in Physics and Astronomy at DigitalCommons@University of Nebraska - Lincoln. It has been accepted for inclusion in Edward Schmidt Publications by an authorized administrator of DigitalCommons@University of Nebraska - Lincoln. 


\author{
FAR-ULTRAVIOLET STELLAR PHOTOMETRY: A FIELD IN MONOCEROS \\ EDWARD G. SCHMIDT \\ Code 4104, E. O. Hulburt Center for Space Research, Naval Research Laboratory, Washington, DC 20375 \\ Internet: eschmidt@unlinfo.unl.edu \\ AND \\ George R. Carruthers \\ Code 4109, E. O. Hulburt Center for Space Research Naval Research Laboratory, Washington, DC 20375 \\ Received 1992 September 8 : accepted 1992 October 27
}

\begin{abstract}
Far-ultraviolet photometry of stars in a field in Monoceros in the wavelength range from $1230 \AA$ to $1600 \AA$ has been carried out using data from an electrographic Schmidt camera carried on a sounding rocket. Ultraviolet magnitudes were extracted for 602 objects in the field. Fifty-eight percent were tentatively identified with visible stars using the SIMBAD data base while another $25 \%$ are blends of objects too close together to separate with our resolution. Eleven of the uv objects coincide with parts of the star clusters NGC 2169, NGC 2244, and NGC 2264 in which individual stars cannot be resolved. As in previous studies, the majority of the identified ultraviolet sources are identified with early-stars. However, there are a significant number for which no such identification was possible, and we suggest that many of these are nearby white dwarfs.
\end{abstract}

Subject headings: open clusters and associations: general - stars: early-type — surveys techniques: photometric - ultraviolet: stars

\section{INTRODUCTION}

Previous papers have reported results from an ongoing farultraviolet survey of various star fields (Carruthers \& Page 1983, 1984a, b, c). This survey has four basic purposes: (1) to detect and provide reference data on a wide variety of hightemperature objects, many of which may later be studied in detail; (2) to provide a historical record of objects which may vary; (3) to provide data for statistical studies; and (4) to search for unusual or unexpected objects. The present paper presents data for a field in Monoceros.

\section{THE OBSERVATIONS}

The data presented in this paper were extracted from three far-ultraviolet electrographic images, obtained by the Naval Research Laboratory in a NASA sounding rocket flight (17.019 DG) on 1982 November 15. The instrument, an electrographic Schmidt camera, was similar to ones used in the Apollo 16 mission (Carruthers 1973) and in previous rocket ultraviolet imagery of the Orion region (Carruthers \& Opal 1977). It had a $\mathrm{KBr}$ photocathode and $\mathrm{a} \mathrm{CaF}_{2}$ corrector plate, yielding a sensitivity range of $1230 \AA-1600 \AA$ (effective wavelength of flat photon flux distribution of $1367 \AA$ ); see Figure 1. The camera had a $75 \mathrm{~mm}$ focal length, $\mathrm{f} / 1$ focal ratio, and a $20^{\circ}$ diameter field of view. Its angular resolution at field center was about 2.5 (with some degradation toward the edges of the field). The electrographic images have a linear density versus exposure relationship to relatively high densities (at least to densities of 1.5) and are not subject to reciprocity failure.

The three images analyzed here, of a field in Monoceros, had exposure times of 4:4, 19:5 and 99:6. All were centered at $\alpha_{1950}=6^{\mathrm{h}} 35^{\mathrm{m}}, \delta_{1950}=9^{\circ} 10^{\prime}$. Figure 2 (Plate 8 ) is a print of the $99: 6$ exposure. During the same flight, similar images were also obtained of a field in the northern part of Orion. Stellar photometry in the Orion region, as well as diffuse-source measurements in Monoceros and Orion, will be discussed in later papers.

\section{THE REDUCTIONS}

The electrographic emulsions were scanned with the NRL PDS microdensitometer. A $16.7 \mu \mathrm{m}$ square sampling aperture was used and samples were spaced at $15 \mu \mathrm{m}$ intervals. These correspond to 0.68 and 0.61 , respectively, on the sky. The complete scan of each image comprised 2048 by 2048 samples.

The IRAF reduction package (Tody 1986) was used to analyze the digitized images. IRAF contains an algorithm which locates stars using the method described by Stetson (1987). This method consists of making a convolution of the image which is equivalent to a least-squares fit of a Gaussian point spread function to a region around each pixel. The convolved image is then searched for peaks which are significantly above the background level. A serious difficulty in the application of this method to the present data arises from the variation of the star image sizes across the field; the FWHM ranges from $\sim 2.5$ at the center to FWHM $\sim 5^{\prime}$ at the edge. In addition, the electrographic frames contain a number of artifacts (scratches, for example) which trigger the star-finding routine. For this reason following the find procedure, each frame was displayed and all the identified star images were verified by eye. Additional stars were also identified which had been missed by the star-finding routine.

Aperture photometry was next performed on all the star images. In view of the variable image structure across the field, a series of apertures were employed which ranged from $6^{\prime}$ to $12^{\prime}$ in radius. The growth curves of the magnitudes with aperture size were then examined and the point at which the curve leveled off was adopted as the stellar magnitude.

For many of the brighter stars, the electrographic images are saturated at the center. Thus the magnitude scale becomes nonlinear. However, due to the variation in image size across the field, the magnitude level at which the nonlinearity sets in depends on location in the field and any correction for it must depend on the distance from the center of the field, $r$. This problem was dealt with by taking advantage of the fact that we 


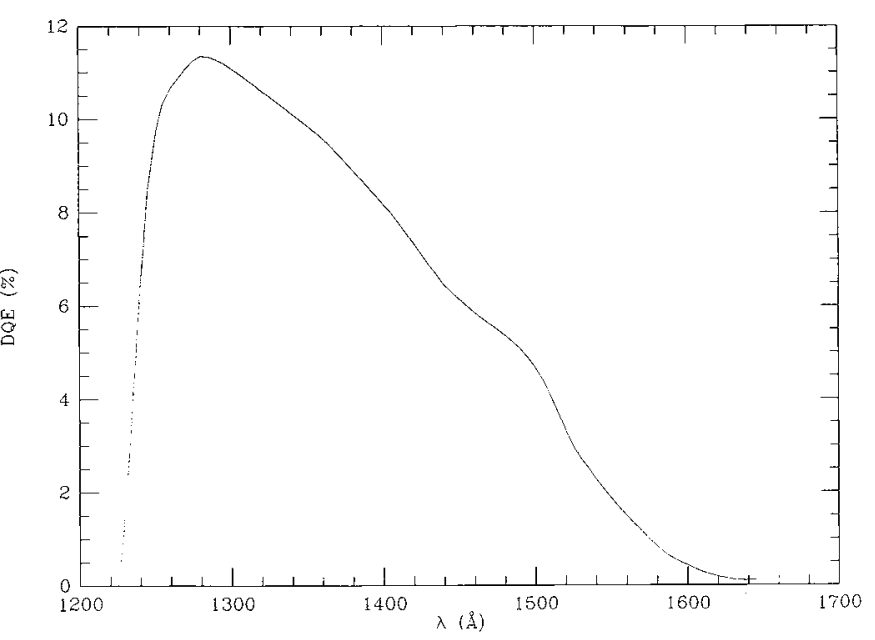

FIG. 1.-Overall detection quantum efficiency as a function of wavelength for the electrographic Schmidt camera used in this project based on laboratory measurements using the camera as a photodiode in comparison with an NBScalibrated photodiode.

have images with different exposure times which saturate at different magnitude levels.

We define the linearity correction, $\delta$, by

$$
\delta\left(r, m_{i}^{\prime}\right)=m_{i}-m_{i}^{\prime},
$$

where $m_{i}^{\prime}$ is the instrumental magnitude measured from exposure $i$ while $m_{i}$ is the instrumental magnitude with the nonlinearity removed. $\delta=0$ if $m_{i}^{\prime}>m_{i}^{s}(r)$ where $m_{i}^{s}(r)$ is the magnitude at which saturation sets in for image $i$ at a location $r$ pixels from the field center. We then assume that

$$
\delta\left(r, m_{i}^{\prime}\right)=\delta\left(0, m_{i}^{\prime}-a r\right) .
$$

This amounts to assuming that the correction curve has the same form at various distances from the image center but slides in magnitude by an amount proportional to $r$.

The adoption of a linear dependence of saturation magnitude on $r$ is not strictly correct. One might consider introducing quadratic or higher terms in $r$ into equation (2) to produce a more realistic correction. However, in practice the linear term removes the effects of saturation to a level below the other errors in the photometry. Thus, the correction is satisfactory, and any higher terms could not be determined sufficiently well to improve the result.

We further assume that $\delta$ is of the same form for all three of the images. If we consider the magnitude differences between exposures $i$ and $j$, assuming $i$ to be the longer exposure, we can write

$$
\delta\left(0, m_{i}^{\prime}-a r\right)-\delta\left(0, m_{j}^{\prime}-a r\right)=\left(m_{i}-m_{j}\right)-\left(m_{i}^{\prime}-m_{j}^{\prime}\right) .
$$

In the regime where frame $j$ is not saturated, $\delta\left(0, m_{j}^{\prime}-a r\right)=0$ and equation (3) becomes

$$
\delta\left(0, m_{i}^{\prime}-a r\right)=m_{j}^{\prime}-m_{i}^{\prime}+\text { const } .
$$

The constant in equation (4) is just the exposure difference between the two images expressed in magnitudes, $-2.5 \log \left(t_{i} / t_{j}\right)$. We then used equation (4) to determine the form of $\delta$. Various values of $a$ were tried and the value which produced the smallest interimage scatter in the present images as well as in a set of images of Orion exposed on the same flight (to be discussed in a separate paper) was adopted. It corresponded to a change in $m_{i}^{s}$ of 2.3 mag between the center and the edge of the field. This is reasonable for the change in the saturation magnitude given the fact that the star images vary by a factor of 4 in area and vary somewhat in shape from field center to edge. It should be noted that stars brighter than about $m_{1367}=5.5$ (on the magnitude scale adopted below) are affected by saturation in even the shortest exposure, and the data for them should be treated with some caution. In the case of the brightest star in the field, 15 Mon, the saturation was so severe that we could not get a reliable value of the magnitude. We have therefore replaced our magnitude with that derived from an $I U E$ spectrum in the manner described below.

All of the instrumental magnitudes were next placed on a linear scale using equation (1). Following this step, the magnitude differences between the three exposures were determined from stars in common, the magnitudes were all placed on a common instrumental system and means were formed for each star. From the scatter among the several exposures, the internal errors were determined. For stars brighter than $m_{1367}=6$ (on the standard magnitude scale adopted below) the rms scatter for a single observation averages $0.14 \mathrm{mag}$. It rises to 0.28 by $m_{1367}=8$.

About 110 objects in our list were identified with stars in the SAO catalog. The celestial coordinates of these stars were fitted to the $x, y$ coordinates in our frames allowing for field rotation, zero point, and a radial distortion. The radial term was relatively small and corresponded to a change in image scale from $40^{\prime} .7 \mathrm{~mm}^{-1}$ at the image center to $38^{\prime} .7 \mathrm{~mm}^{-1}$ at the edge. It was fitted with a second-order polynomial. The scatter of the SAO stars about the fitted solution was 3.8, on the order of the size of the star images. The coordinates of the stars derived from our images are listed in columns (2) and (3) of Table 1 .

The instrumental magnitudes were calibrated to a standard system using data from the $I U E$ archives. We found 11 earlytype stars with $I U E$ spectra which could be identified uniquely with stars in our list. A number of stars were rejected because several IUE stars were too close together to be distinct on our images. Several were also rejected due to discrepancies between our magnitudes and those from IUE. These latter are likely to be caused by confusion in identification due to our rather poor spatial resolution.

The IUE spectrum was convolved with our instrumental response function (Fig. 1) for each of the calibration stars and the resulting flux was converted to a magnitude on a system in which $m_{1367}=11$ corresponds to $F_{1367}=3.6 \times 10^{-13} \mathrm{ergs}$ $\mathrm{cm}^{-2} \mathrm{~s}^{-1} \AA^{-1}$. The differences between our instrumental magnitudes and the $I U E$ values had an $\mathrm{rms}$ scatter of $0.81 \mathrm{mag}$. With 11 calibrating stars, our zero point has a standard error of $0.24 \mathrm{mag}$. A plot of the differences between the instrumental and the IUE magnitudes showed no trend with brightness. This indicates again that nonlinearities have been dealt with adequately. The fourth column in Table 1 lists our ultraviolet magnitudes.

\section{STAR IDENTIFICATIONS}

We searched a region of $10^{\prime}$ radius around each uv object in the SIMBAD data base (see Egret, Wenger, \& Dubois 1991 for a description of SIMBAD and further references). The resulting identifications are listed in the fifth column of Table 1. In making identifications, the resolution of our images created some difficulty. Often several stars of similar brightness fall within the circle of confusion for a given uv object. Such cases are indicated by the word "Blend" in column (5). In deciding 
TABLE 1

Objects Detected in The Ultraviolet

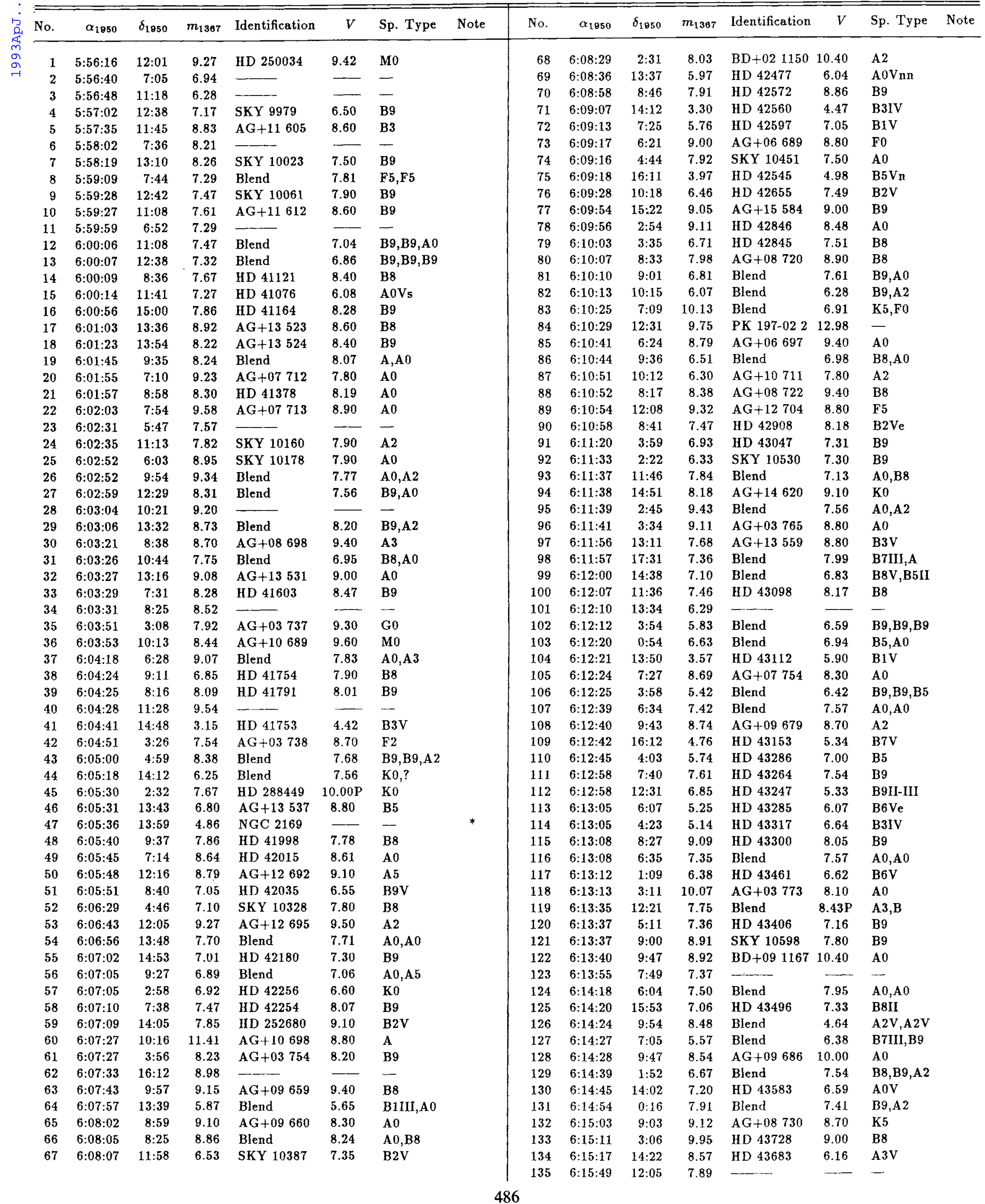


TABLE $1-$ Continued

\begin{tabular}{|c|c|c|c|c|c|c|c|c|c|c|c|c|c|c|c|}
\hline No. & $\alpha_{1850}$ & $\delta_{1050}$ & $m_{1367}$ & Identification & $V$ & Sp. Type & Note & No. & $\alpha_{1850}$ & $\delta_{1050}$ & $m_{1387}$ & Identification & $V$ & Sp. Type & Note \\
\hline 136 & $6: 15: 56$ & $9: 14$ & 9.09 & $\mathrm{BD}+091183$ & 9.30 & - & & 203 & $6: 23: 09$ & $4: 00$ & 9.49 & & - & - & \\
\hline 137 & $6: 16: 03$ & $11: 08$ & 8.04 & Blend & 6.90 & $\mathrm{~A} 0, \mathrm{~A}$ & & 204 & $6: 23: 16$ & $12: 12$ & 7.89 & Blend & 8.03 & $\mathrm{B5}_{\mathbf{1}} \mathrm{A} 2$ & \\
\hline 138 & $6: 16: 10$ & $2: 35$ & 9.02 & HD 43889 & 9.40 & $\mathrm{~B} 8$ & & 205 & $6: 23: 17$ & $5: 01$ & 9.14 & $\mathrm{AG}+04756$ & 9.10 & $\mathrm{~A} 2$ & \\
\hline 139 & $6: 16: 19$ & $12: 43$ & 6.32 & Blend & 6.28 & $\mathbf{B 8}, \mathbf{A 0}$ & & 206 & $6: 23: 26$ & $2: 27$ & 7.29 & HD 45137 & 6.51 & $\mathrm{AOV}$ & \\
\hline 140 & $6: 16: 21$ & $17: 25$ & 7.00 & SKY 10714 & 6.32 & B9IIIsp. & & 207 & $6: 23: 42$ & $-0: 40$ & 7.61 & SKY 11051 & 6.78 & $\mathrm{~B} 9$ & \\
\hline 141 & $6: 16: 28$ & $4: 18$ & 8.61 & SKY 10710 & 7.50 & A0 & & 208 & $6: 23: 44$ & $15: 33$ & 7.51 & IHD 45180 & 6.88 & B9V & \\
\hline 142 & $6: 16: 38$ & $6: 10$ & 7.89 & HD 439.12 & 8.26 & A0 & & 209 & $6: 23: 48$ & $11: 39$ & 9.68 & $A G+11683$ & 8.80 & B8 & \\
\hline 143 & $6: 16: 53$ & $12: 16$ & 7.26 & SKY 10753 & 7.70 & B8 & & 210 & $6: 23: 49$ & $4: 35$ & 6.82 & HD 45136 & 7.97 & A 0 & \\
\hline 144 & $6: 16: 57$ & $10: 58$ & 7.16 & HD 43984 & 8.30 & B8 & & 211 & $6: 23: 57$ & $8: 03$ & 7.52 & HD 45166 & 9.88 & Bpevar & \\
\hline 145 & $6: 17: 12$ & $10: 48$ & 7.74 & $A G+10727$ & 9.00 & AO & & 212 & $6: 24: 01$ & $16: 53$ & 9.00 & LS 57 & $10.90 \mathrm{P}$ & B... & \\
\hline 146 & $6: 17: 22$ & $4: 45$ & 7.54 & HD 44052 & 8.40 & B8 & & 213 & $6: 24: 12$ & $10: 56$ & 7.70 & Blend & 8.04 & B8,B3 & \\
\hline 147 & $6: 17: 31$ & $11: 57$ & 8.05 & $A G+12730$ & 9.00 & $\mathbf{A}$ & & 214 & $6: 24: 13$ & $9: 07$ & 9.55 & Blend & 7.41 & $A 2, \mathbf{A} 0$ & \\
\hline 148 & $6: 17: 37$ & $13: 04$ & 9.61 & $A G+13574$ & 8.90 & B9III & & 215 & $6: 24: 13$ & $4: 15$ & 8.89 & $A G+04767$ & 9.70 & AO & \\
\hline 149 & $6: 17: 51$ & $7: 45$ & 6.78 & HD 44109 & 6.76 & B9 & & 216 & $6: 24: 21$ & $14: 55$ & 5.93 & $\mathrm{HD} 45314$ & 6.64 & O9:pe & \\
\hline 150 & $6: 18: 06$ & $11: 44$ & 6.02 & HD 44173 & 6.53 & B5III & & 217 & $6: 24: 25$ & $1: 00$ & 7.62 & HD 45357 & 6.71 & AIVn & \\
\hline 151 & $6: 18: 07$ & $8: 58$ & 8.48 & Blend & 8.04 & $\mathbf{A} 0, \mathbf{A} 0$ & & 218 & $6: 24: 33$ & $8: 10$ & 7.59 & Blend & 9.08 & $?, \mathrm{G} 5$ & \\
\hline 152 & $6: 18: 08$ & $17: 27$ & 9.08 & $\mathbf{A G}+17605$ & 8.20 & AO & & 219 & $6: 24: 33$ & $4: 02$ & 9.25 & LS VI +034 & $11.80 \mathrm{P}$ & $\mathbf{B}$ & \\
\hline 153 & $6: 18: 11$ & $14: 42$ & 6.35 & HD 44172 & 7.34 & $\mathrm{~B} 6 \mathrm{~V}$ & & 220 & $6: 24: 35$ & $0: 16$ & 7.39 & Blend & $7.65 \mathrm{P}$ & $\mathrm{B} 8, \mathrm{~A} 0, \mathrm{~A}$ & \\
\hline 154 & $6: 18: 27$ & $9: 45$ & 6.20 & HD 44235 & 7.99 & B8 & & 221 & $6: 24: 47$ & $-0: 32$ & 8.84 & Blend & 7.84 & $A 5, \mathbf{A} 5$ & \\
\hline 155 & $6: 18: 31$ & $8: 27$ & 9.03 & - & $\rightarrow-$ & - & & 222 & $6: 24: 58$ & $8: 29$ & 9.07 & Blend & 7.90 & $\mathbf{A} 0, \mathbf{A} 2$ & \\
\hline 156 & $6: 19: 07$ & $14: 17$ & 7.87 & HD 44351 & 8.27 & $\mathbf{A}$ & & 223 & $6: 25: 01$ & $15: 44$ & 9.48 & $A G+15620$ & 8.10 & $\mathrm{~B} 9$ & \\
\hline 157 & $6: 19: 14$ & $8: 46$ & 8.99 & Blend & 7.74 & $\mathrm{~A} 0, \mathrm{~A} 2, \mathrm{~A} 0$ & & 224 & $6: 25: 07$ & $7: 03$ & 9.06 & Blend & 7.90 & $\mathrm{~A} 0, \mathrm{~B} 8$ & \\
\hline 158 & $6: 19: 15$ & $10: 57$ & 7.03 & $\mathrm{HD} 44373$ & 8.50 & $\mathrm{~B} 8$ & & 225 & $6: 25: 08$ & $12: 48$ & 8.69 & - - - & - & - & \\
\hline 159 & $6: 19: 23$ & $12: 01$ & 8.22 & $A G+12733$ & 8.50 & A0 & & 226 & $6: 25: 22$ & $5: 20$ & 9.49 & HD 45530 & 7.41 & Alp & \\
\hline 160 & $6: 19: 26$ & $2: 14$ & 9.71 & HD 44333 & 6.31 & A4.5V & & 227 & $6: 25: 33$ & $5: 42$ & 9.65 & - - - & $\longrightarrow$ & - & \\
\hline 161 & $6: 19: 53$ & $8: 21$ & 7.76 & HD 44498 & 8.83 & $\mathrm{B2} .5 \mathrm{~V}$ & & 228 & $6: 25: 35$ & $11: 09$ & 8.48 & $A G+11688$ & 9.70 & A5 & \\
\hline 162 & $6: 20: 02$ & $12: 21$ & 9.86 & $A G+12735$ & 8.30 & A0 & & 229 & $6: 25: 36$ & $0: 16$ & 8.09 & Blend & $10.00 \mathrm{P}$ & $\mathbf{A} 2, \mathbf{A}$ & \\
\hline 163 & $6: 20: 15$ & $6: 33$ & 9.79 & $A G+06728$ & 8.60 & AO & & 230 & $6: 25: 40$ & $7: 14$ & 8.52 & HD 257779 & $\mathbf{8 . 8 3}$ & B8 & \\
\hline 164 & $6: 20: 18$ & $17: 39$ & 8.82 & HD 44496 & 6.93 & A0 & & 231 & $6: 25: 52$ & $11: 21$ & 7.97 & HD 257971 & 8.90 & B0.5III & \\
\hline 165 & $6: 20: 23$ & $15: 52$ & 8.52 & Blend & 7.73 & B7V,B9p & & 232 & $6: 25: 54$ & $8: 02$ & 9.03 & - - & & - & \\
\hline 166 & $6: 20: 23$ & $16: 34$ & 8.30 & HD 44584 & 8.01 & $\mathrm{Bg}$ & & 233 & $6: 26: 00$ & $14: 07$ & 7.57 & $\mathrm{AG}+14659$ & 8.70 & F5 & \\
\hline 167 & $6: 20: 28$ & $5: 14$ & 8.63 & Blend & 8.04 & $\mathrm{~B} 9, \mathrm{~A} 2$ & & 234 & $6: 26: 02$ & $2: 07$ & 6.78 & Blend & $8.18 \mathrm{P}$ & $\mathbf{B} 9, \mathbf{A}, \mathbf{A} 7$ & \\
\hline 168 & $6: 20: 28$ & $0: 17$ & 9.37 & HD 44718 & 8.73 & A0 & & 235 & $6: 26: 10$ & $5: 26$ & 8.99 & $A G+05798$ & 10.70 & Fo & \\
\hline 169 & $6: 20: 32$ & $15: 06$ & 8.82 & HD 44637 & 8.03 & B2V:pe & & 236 & $6: 26: 20$ & $0: 16$ & 7.43 & SKY 11161 & 6.70 & $\mathrm{~A} 0, \mathrm{~A}$ & \\
\hline 170 & $6: 20: 34$ & $9: 17$ & 8.64 & $A G+09701$ & 8.50 & Fo & & 237 & $6: 26: 26$ & $13: 40$ & 7.75 & $\mathrm{AG}+13594$ & 8.40 & B 9 & \\
\hline 171 & $6: 20: 34$ & $6: 47$ & 8.98 & Blend & 9.18 & $\mathrm{~B} 9, \mathrm{~A} 0$ & & 238 & $6: 26: 36$ & $2: 20$ & 9.07 & Blend & $8.97 \mathrm{P}$ & $\mathbf{A 0 , A 5}$ & \\
\hline 172 & $6: 20: 37$ & $8: 56$ & 8.18 & $A G+08751$ & 9.30 & $\mathrm{B8}$ & & 239 & $6: 27: 16$ & 18:02 & 8.88 & Blend & 7.30 & $\mathbf{A} 0, \mathbf{A 2}$ & \\
\hline 173 & $6: 20: 49$ & $15: 24$ & 5.17 & $+\frac{1}{-2}$ & $\cdots$ & - & & 240 & $6: 27: 18$ & $7: 52$ & 7.30 & HD 45802 & - - & A & \\
\hline 174 & $6: 20: 53$ & $10: 56$ & 8.52 & HD 44712 & 7.76 & A0 & & 241 & $6: 27: 23$ & $8: 32$ & 8.14 & SKY 11187 & 7.50 & F8 & \\
\hline 175 & $6: 20: 58$ & $8: 04$ & 8.88 & ——— & - - & - & & 242 & $6: 27: 26$ & $4: 27$ & 8.12 & NGC 2244 & - & B2IV-V,B & $*$ \\
\hline 176 & $6: 20: 59$ & $14: 06$ & 9.07 & $\mathrm{AG}+14644$ & 7.95 & $\mathrm{~A} 2 \mathrm{Ib}$ & & 243 & $6: 27: 36$ & $4: 40$ & 8.02 & NGC 2244 & - & - & $*$ \\
\hline 177 & $6: 21: 01$ & $3: 55$ & 5.06 & HD 44700 & 6.40 & B3V & & 244 & $6: 27: 37$ & $9: 08$ & 8.60 & HD 45827 & 6.57 & A0III & \\
\hline 178 & $6: 21: 03$ & $0: 04$ & 8.25 & Blend & 7.63 & $\mathrm{~A} 0, \mathrm{~A} 2, \mathrm{~A} 0$ & & 245 & $6: 27: 38$ & $8: 24$ & 8.14 & $\mathrm{AG}+08779$ & 8.80 & A & \\
\hline 179 & $6: 21: 04$ & $7: 33$ & 9.66 & Blend & 8.81 & $\mathrm{~A} 0, \mathrm{AO}$ & & 246 & $6: 27: 45$ & $7: 15$ & 5.71 & HD 45789 & 7.10 & B2.5IV-V & \\
\hline 180 & $6: 21: 30$ & $9: 12$ & 7.01 & HD 44782 & 7.38 & $\mathrm{~B} 9$ & & 247 & $6: 27: 59$ & $7: 02$ & 6.99 & HD 258491 & 8.87 & $\mathrm{~K} 7$ & \\
\hline 181 & $6: 21: 33$ & $8: 55$ & 6.58 & HD 44783 & 6.27 & B8Vn & & 248 & $6: 28: 00$ & $8: 46$ & 9.16 & $\mathrm{AG}+08781$ & 8.40 & A 0 & \\
\hline 182 & $6: 21: 34$ & $8: 21$ & 8.93 & Blend & 7.16 & A5,B2IV: & & 249 & $6: 28: 06$ & $8: 01$ & 9.25 & IRC +10125 & 8.60 & & \\
\hline 183 & $6: 21: 36$ & $8: 01$ & 8.97 & HD 44813 & 8.27 & A3 & & 250 & $6: 28: 11$ & $10: 08$ & 6.55 & Blend & 6.97 & $\mathrm{~B} 8, \mathrm{~A} 2, \mathrm{~B} 7$ & \\
\hline 184 & $6: 21: 39$ & $13: 29$ & 8.94 & $-\ldots$ & - & - & & 251 & $6: 28: 14$ & $3: 04$ & 8.46 & HD 45912 & 8.80 & F8 & \\
\hline 185 & $6: 21: 38$ & $12: 17$ & 9.84 & $A G+12738$ & 8.80 & A0 & & 252 & $6: 28: 23$ & $11: 20$ & 5.02 & HD 45995 & 6.03 & B2V:nne & \\
\hline 186 & $6: 21: 59$ & $13: 54$ & 8.81 & $B D+131236$ & 9.10 & B6IV & & 253 & $6: 28: 28$ & $7: 34$ & 9.12 & HD 45930 & 8.66 & A 0 & \\
\hline 187 & $6: 22: 00$ & $15: 01$ & 8.75 & Blend & 8.14 & B3V,B9V & & 254 & $6: 28: 30$ & $6: 02$ & 6.90 & CL Collinde & 5.40 & & \\
\hline 188 & $6: 22: 10$ & $5: 26$ & 8.62 & Blend & 8.16 & $\mathbf{A} 0, \mathbf{A} 0$ & & 255 & $6: 28: 31$ & $4: 32$ & 5.83 & NGC 2244 & $\longrightarrow$ & A6V,A0II & $*$ \\
\hline 189 & $6: 22: 15$ & $14: 53$ & 8.76 & $\mathrm{AG}+14646$ & 8.80 & B3V & & 256 & $6: 28: 32$ & $9: 57$ & 5.97 & Blend & 7.26 & B8,B3,BIII & \\
\hline 190 & $6: 22: 19$ & $17: 05$ & 7.46 & HD 44904 & 6.99 & $\mathrm{~B} 9$ & & 257 & $6: 28: 40$ & $8: 36$ & 8.69 & $A G+08783$ & 9.30 & B8 & \\
\hline 191 & $6: 22: 19$ & $4: 20$ & 7.41 & HD 44907 & 7.30 & B9 & & 258 & $6: 28: 48$ & $11: 52$ & 6.09 & HD 46075 & 6.65 & B6III & \\
\hline 192 & $6: 22: 25$ & $11: 41$ & 9.24 & HD 44965 & 7.82 & B3II & & 259 & $6: 29: 02$ & $2: 56$ & 9.40 & Blend & $8.91 \mathrm{P}$ & $\mathbf{B 9}, \mathbf{A} 3$ & \\
\hline 193 & $6: 22: 32$ & $8: 26$ & 10.11 & $\mathrm{AG}+08757$ & 10.30 & B8 & & 260 & $6: 29: 03$ & $4: 51$ & 7.43 & NGC 2244 & - & - & $*$ \\
\hline 194 & $6: 22: 37$ & $10: 49$ & 7.16 & Blend & 7.31 & $\mathrm{B8}, \mathrm{B8}$ & & 261 & $6: 29: 07$ & $15: 11$ & 8.92 & HD 46121 & 8.36 & A0 & \\
\hline 195 & $6: 22: 38$ & $12: 15$ & 7.90 & $A G+12742$ & 9.00 & A2 & & 262 & $6: 29: 35$ & $6: 21$ & 7.09 & $-\ldots$ & - & - & \\
\hline 196 & $6: 22: 45$ & $13: 16$ & 8.48 & $A G+13586$ & 8.40 & B8V & & 263 & $6: 29: 38$ & $2: 15$ & 7.35 & Blend & $8.50 \mathrm{P}$ & $\mathrm{K} 0, \mathrm{~K} 5, \mathrm{~F} 0$ & \\
\hline 197 & $6: 22: 47$ & $14: 05$ & 8.17 & $A G+14649$ & 8.60 & $\mathrm{~A} 2 \mathrm{~V}$ & & 264 & $6: 29: 40$ & $5: 57$ & 7.46 & HD 46179 & 6.69 & B9V & \\
\hline 198 & $6: 22: 48$ & $1: 40$ & 6.84 & HD 45050 & 6.66 & B9V & & 265 & $6: 29: 43$ & $17: 47$ & 8.57 & HD 46162 & 8.48 & AO & \\
\hline 199 & $6: 22: 54$ & $8: 05$ & 8.12 & Blend & 8.23 & $\mathrm{A0}, \mathrm{B8}$ & & 266 & $6: 29: 46$ & $9: 16$ & 8.40 & $\mathrm{BD}+091274$ & 9.20 & $?$ & \\
\hline 200 & $6: 22: 54$ & $7: 42$ & 9.40 & Blend & 8.45 & $\mathbf{A} 0, \mathbf{A} 0$ & & 267 & $6: 30: 00$ & $5: 10$ & 4.99 & NGC 2244 & $\cdots$ & $\mathrm{B8}$ & $*$ \\
\hline 201 & $6: 22: 56$ & $11: 10$ & 8.85 & Blend & 7.15 & $\mathbf{A} 0, \mathbf{A} 0$ & & 268 & $6: 30: 05$ & $6: 13$ & 7.08 & Blend & $10.24 \mathrm{P}$ & F8,? & \\
\hline 202 & $6: 23: 03$ & $8: 12$ & 8.12 & $\mathrm{BD}+081325$ & 9.20 & $\mathrm{B8}$ & & $\begin{array}{l}269 \\
270\end{array}$ & $\begin{array}{l}6: 30: 07 \\
6: 30: 12\end{array}$ & $\begin{array}{r}5: 44 \\
17: 05\end{array}$ & $\begin{array}{l}9.03 \\
7.26\end{array}$ & $\begin{array}{l}\text { HD } 259440 \\
\text { Blend }\end{array}$ & $\begin{array}{l}9.17 \\
6.96\end{array}$ & $\begin{array}{l}\text { B0pe } \\
\text { A0,Be }\end{array}$ & \\
\hline
\end{tabular}




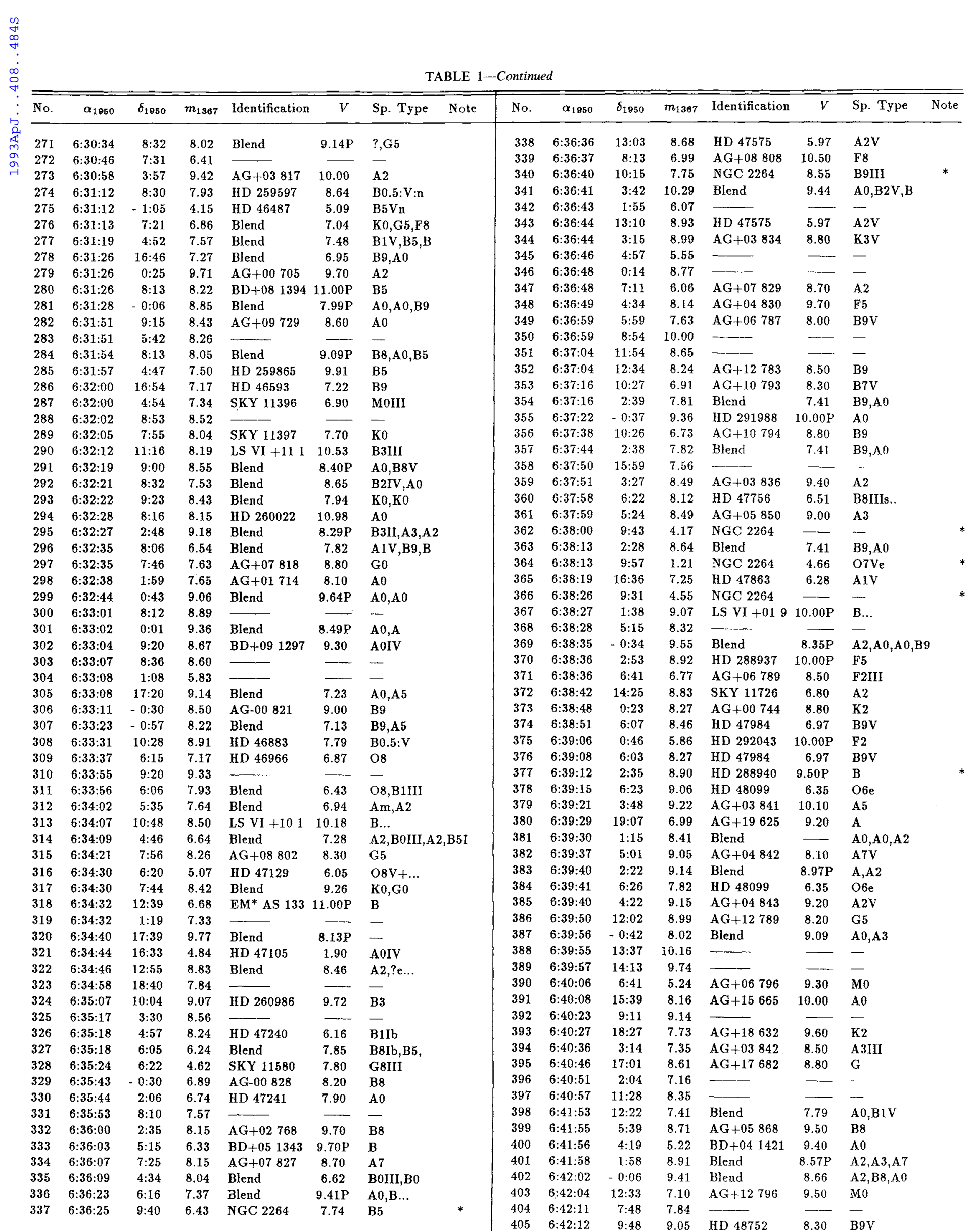


TABLE $1-$ Continued

\begin{tabular}{|c|c|c|c|c|c|c|c|c|c|c|c|c|c|c|}
\hline No. & $\alpha_{1850}$ & $\delta_{1850}$ & $m_{1 \mathbf{3} 67}$ & Identification & V & Sp. Type & No. & $\alpha_{1850}$ & $\delta_{1950}$ & $m_{1387}$ & Identification & $V$ & Sp. Type & Note \\
\hline 406 & $6: 42: 15$ & $6: 35$ & 8.85 & $A G+06805 \quad 1$ & 10.00 & A2 & 473 & $6: 49: 21$ & $13: 43$ & 9.87 & $A G+13648$ & 9.10 & $\mathrm{~B} 0$ & \\
\hline 407 & $6: 42: 23$ & $2: 46$ & 9.13 & Blend & $9.03 \mathrm{P}$ & B..., $\mathrm{A} 0$ & 474 & $6: 49: 30$ & $11: 46$ & 9.40 & SKY 12172 & 7.60 & KO & \\
\hline 408 & $6: 42: 36$ & $15: 34$ & 8.04 & $A G+15670$ & 8.20 & B9 & 475 & $6: 49: 39$ & $7: 36$ & 8.93 & $A G+07877$ & 9.30 & $\mathrm{~K} 2$ & \\
\hline 409 & $6: 42: 42$ & $0: 54$ & 7.19 & Blend & $-\ldots$ & $\mathrm{A} 2, \mathbf{A} 3$ & 476 & $6: 49: 55$ & $15: 09$ & 8.84 & $A G+15694$ & 8.50 & F8 & \\
\hline 410 & $6: 42: 43$ & $10: 02$ & 8.20 & Blend & 10.23 & $\mathrm{~A} 2, \mathrm{~B} \ldots$ & 477 & $6: 49: 56$ & $1: 38$ & 8.40 & - & - & - & \\
\hline 411 & $6: 43: 02$ & $9: 12$ & 7.80 & - - & - & - & 478 & $6: 49: 59$ & $3: 31$ & 9.04 & - - & - & - & \\
\hline 412 & $6: 43: 05$ & $4: 05$ & 8.04 & - - & {[} & - & 479 & $6: 50: 00$ & $6: 05$ & 9.38 & $A G+05898$ & 9.40 & A0 & \\
\hline 413 & $6: 43: 05$ & $4: 47$ & 8.94 & SKY 11877 & 7.70 & $\mathbf{A} 0$ & 480 & $6: 50: 00$ & $0: 45$ & 7.37 & Blend & $8.74 \mathrm{P}$ & $\mathbf{A} 0, \mathbf{A}, \mathbf{A} \mathbf{0}$ & \\
\hline 414 & $6: 43: 10$ & $-0: 49$ & 7.35 & SKY 11883 & 6.70 & AO & 481 & $6: 50: 01$ & $5: 35$ & 6.16 & & - & - & \\
\hline 415 & $6: 43: 13$ & $0: 12$ & 8.43 & Blend & 8.48 & $\mathrm{~A} 0, \mathrm{~A} 0$ & 482 & $6: 50: 14$ & $16: 13$ & 7.59 & Blend & 9.14 & $\mathrm{~B} 0, \mathrm{~K} 0$ & \\
\hline 416 & $6: 43: 21$ & $9: 27$ & 7.70 & - & - & - & 483 & $6: 50: 27$ & $-0: 01$ & 8.39 & Blend & 8.96 & $\mathrm{~B} 8, \mathrm{~B} 5, \mathrm{~A} 0 \mathrm{~V}, \mathrm{~A} 2$ & \\
\hline 417 & $6: 43: 27$ & $5: 37$ & 8.51 & - - & - & - & 484 & $6: 50: 27$ & $4: 35$ & 9.90 & $\mathrm{AG}+04875$ & 9.00 & $\mathrm{~K} 2$ & \\
\hline 418 & $6: 43: 31$ & $6: 15$ & 8.52 & - — & $\ldots$ & - & 485 & $6: 50: 30$ & $8: 20$ & 7.42 & HD 50277 & 5.80 & FOVn & \\
\hline 419 & $6: 43: 48$ & $4: 48$ & 8.48 & $A G+04857$ & 8.40 & Ko & 486 & $6: 50: 34$ & $11: 04$ & 7.15 & Blend & 6.01 & K0III,G0 & \\
\hline 420 & $6: 43: 50$ & 5:01 & 8.46 & $A G+05872$ & 9.20 & A2 & 487 & $6: 50: 53$ & $6: 17$ & 9.75 & $\mathrm{AG}+06825$ & 10.10 & G0 & \\
\hline 421 & $6: 43: 54$ & $-0: 29$ & 7.67 & Blend & $9.50 \mathrm{P}$ & $A m, B 9$ & 488 & $6: 50: 54$ & $1: 28$ & 8.05 & Blend & 7.54 & $\mathrm{B9}, \mathrm{A3}$ & \\
\hline 422 & $6: 44: 03$ & $11: 06$ & 7.44 & $A G+11739$ & 8.10 & A5 & 489 & $6: 51: 15$ & $4: 29$ & 9.09 & $\mathrm{AG}+04880$ & 9.10 & G0 & \\
\hline 423 & $6: 44: 06$ & $2: 54$ & 6.72 & Blend & $8.45 \mathrm{P}$ & $\mathrm{A}, \mathrm{A} 0, \mathrm{~B} 9$ & 490 & $6: 51: 21$ & $15: 02$ & 7.67 & - - & - & - & \\
\hline 424 & $6: 44: 07$ & $17: 26$ & 7.18 & $A G+17690$ & 8.50 & $\mathrm{~K} 0$ & 491 & $6: 51: 27$ & $5: 47$ & 9.40 & Blend & 7.94 & $\mathrm{~B} 9, \mathrm{~A} 0$ & \\
\hline 425 & $6: 44: 18$ & $8: 59$ & 4.64 & Blend & 8.04 & A7V,G0 & 492 & $6: 51: 29$ & $15: 50$ & 8.99 & SKY 12268 & 6.50 & G8III & \\
\hline 426 & $6: 44: 20$ & $1: 04$ & 8.07 & HD 289117 & - & B8 & 493 & $6: 51: 43$ & $11: 32$ & 8.28 & $A G+11761$ & 9.20 & B9 & \\
\hline 427 & $6: 44: 26$ & $3: 03$ & 6.89 & - & - & - & 494 & $6: 51: 58$ & $9: 58$ & 7.48 & - & - & - & \\
\hline 428 & $6: 44: 29$ & $0: 23$ & 8.68 & Blend & 9.80 & $\mathrm{~A} 0, \mathrm{~A} 7$ & 495 & $6: 52: 03$ & $7: 21$ & 7.92 & Blend & 7.88 & $\mathrm{~A} 2, \mathrm{~A} 3$ & \\
\hline 429 & $6: 44: 35$ & $8: 18$ & 8.29 & 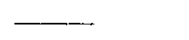 & - & - & 496 & $6: 52: 06$ & $1: 49$ & 8.65 & Blend & - & $\mathrm{A} 5, \mathrm{~A} 9, \mathrm{~A} 0, \mathrm{~A} 3$ & \\
\hline 430 & $6: 44: 40$ & $7: 00$ & 6.75 & 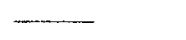 & $-\cdots$ & - & 497 & $6: 52: 08$ & $3: 19$ & 9.32 & HD 50794 & 8.30 & Fo & \\
\hline 431 & $6: 44: 50$ & $14: 35$ & 8.87 & Blend & 7.39 & $\mathbf{A} 0, \mathbf{A} 2$ & 498 & $6: 52: 09$ & $6: 01$ & 8.61 & Blend & 7.63 & F8,G5 & \\
\hline 432 & $6: 45: 07$ & $14: 54$ & 7.59 & Blend & 7.91 & $\mathrm{~K} 0, \mathrm{~K} 7$ & 499 & $6: 52: 13$ & $-0: 01$ & 7.71 & Blend & $9.65 \mathrm{P}$ & $\mathrm{A}, \mathrm{A} 2 \mathrm{~V}$ & \\
\hline 433 & $6: 45: 08$ & $1: 54$ & 9.17 & Blend & - & $\mathrm{B8}, \mathrm{B9}, \mathrm{B} 9$ & 500 & $6: 52: 13$ & $14: 13$ & 8.87 & $\mathrm{AG}+14721$ & 8.50 & $\mathrm{~K}$ & \\
\hline 434 & $6: 45: 08$ & $11: 51$ & 8.90 & $A G+11741$ & 9.40 & Mo & 501 & $6: 52: 15$ & $7: 14$ & 7.77 & Blend & 8.07 & $\mathbf{A} 0, \mathbf{A 3}$ & \\
\hline 435 & $6: 45: 18$ & $-0: 47$ & 9.25 & Blend & $8.23 \mathrm{P}$ & $\mathrm{B} 8, \mathrm{~A} 3, \mathrm{~A} 2$ & 502 & $6: 52: 32$ & $5: 39$ & 7.85 & Blend & $7.54 \mathrm{P}$ & B2Vne,B & \\
\hline 436 & $6: 45: 39$ & $8: 27$ & 8.80 & $A G+08842$ & 9.00 & F8 & 503 & $6: 52: 33$ & $6: 12$ & 8.44 & $A G+06834$ & 9.00 & F0 & \\
\hline 437 & $6: 45: 40$ & $-0: 30$ & 5.66 & Blend & 8.65 & $\mathrm{~A} 5, \mathrm{~A} 0, \mathrm{~A} 0$ & 504 & $6: 52: 38$ & $7: 01$ & 9.76 & $\mathbf{A G}+06832$ & 8.70 & $\mathbf{K} 0$ & \\
\hline 438 & $6: 45: 44$ & $2: 27$ & 7.32 & $\ldots$ & $\ldots$ & - & 505 & $6: 52: 44$ & $0: 30$ & 8.14 & Blend & $7.80 \mathrm{P}$ & - & * \\
\hline 439 & $6: 45: 53$ & $6: 44$ & 7.99 & $\mathrm{BD}+061390$ & $9.50 \mathrm{P}$ & - & 506 & $6: 52: 45$ & $14: 19$ & 8.70 & $A G+14721$ & 8.50 & $\mathbf{K}$ & \\
\hline 440 & $6: 45: 58$ & $4: 16$ & 9.18 & $\ldots$ & $-\cdots$ & - & 507 & $6: 52: 51$ & $8: 01$ & 7.61 & Blend & 8.19 & $\mathbf{A}, \mathbf{A} 2$ & \\
\hline 441 & $6: 45: 59$ & $1: 44$ & 8.52 & Blend & $8.19 \mathrm{P}$ & $\mathrm{B} 8, \mathrm{~B} 8 \mathrm{~V}, \mathrm{B8}, \mathrm{B8}$ & 508 & $6: 52: 59$ & $3: 13$ & 9.86 & - & - & - & \\
\hline 442 & $6: 46: 06$ & $17: 25$ & 8.33 & Blend & 7.54 & $\mathrm{~K} 0, \mathrm{~F} 8$ & 509 & $6: 53: 10$ & $10: 00$ & 8.59 & HD 51104 & 5.92 & B8Vn & \\
\hline 443 & $6: 46: 09$ & $5: 07$ & 8.58 & $A G+05881$ & 9.30 & $\mathrm{~B} 8$ & 510 & $6: 53: 19$ & $8: 52$ & 8.29 & SKY 12314 & 7.40 & F8Ib: & \\
\hline 444 & $6: 46: 11$ & $9: 03$ & 9.09 & SKY 12009 & 6.90 & Ko & 511 & $6: 53: 27$ & $5: 59$ & 6.07 & $A G+06837$ & 8.40 & B8 & \\
\hline 445 & $6: 46: 28$ & $8: 23$ & 8.99 & $A G+08847$ & 8.10 & F5 & 512 & $6: 53: 27$ & $9: 26$ & 7.87 & $A G+09798$ & 9.00 & F0 & \\
\hline 446 & $6: 46: 48$ & $14: 08$ & 9.13 & - & $\cdots$ & - & 513 & $6: 53: 50$ & $6: 46$ & 9.10 & $A G+06838$ & 9.00 & A & \\
\hline 447 & $6: 46: 48$ & $16: 32$ & 5.57 & $\mathrm{AG}+16679$ & 9.20 & A0 & 514 & $6: 53: 55$ & $10: 30$ & 5.62 & Blend & 8.20 & G0,G5 & \\
\hline 448 & $6: 46: 58$ & $4: 60$ & 9.44 & $\ldots$ & - & - & 515 & $6: 54: 02$ & $11: 54$ & 7.80 & Blend & 6.06 & F2Ib-II, & \\
\hline 449 & $6: 47: 04$ & $1: 55$ & 8.76 & $\mathrm{AG}+01765$ & 9.50 & B8 & 516 & $6: 54: 04$ & $10: 19$ & 6.08 & $A G+10852$ & 8.70 & G0 & \\
\hline 450 & $6: 47: 18$ & $1: 22$ & 5.12 & Blend & - & - & 517 & $6: 54: 32$ & $6: 34$ & 8.64 & $A G+06841$ & 9.60 & A0 & \\
\hline 451 & $6: 47: 21$ & $5: 06$ & 5.37 & - - & - & - & 518 & $6: 54: 36$ & $9: 50$ & 9.44 & $A G+09805$ & 8.60 & A5 & \\
\hline 452 & $6: 47: 25$ & $0: 24$ & 8.24 & Blend & $8.71 \mathrm{P}$ & $\mathrm{O} 8, \mathrm{~A} 0, \mathrm{~A} 0, \mathrm{~A} 0, \mathrm{~A} 0, \mathrm{~B}$ & 519 & $6: 54: 42$ & $4: 07$ & 8.04 & $\ldots$ & - & - & \\
\hline 453 & $6: 47: 25$ & $7: 34$ & 9.09 & - & - & - & 520 & $6: 54: 45$ & $3: 42$ & 9.71 & $A G+03891$ & 9.50 & A7 & \\
\hline 454 & $6: 47: 35$ & $4: 47$ & 10.06 & $A G+04868$ & 9.00 & $\mathbf{A}$ & 521 & $6: 54: 47$ & $7: 05$ & 7.91 & $\mathrm{BD}+071528$ & $11.00 \mathrm{P}$ & $\mathrm{B} \ldots$ & \\
\hline 455 & $6: 47: 38$ & $0: 00$ & 8.19 & HD 292409 & 11.62 & $\mathrm{~A} 0 \mathrm{~V}$ & $\mathbf{5 2 2}$ & $6: 54: 56$ & $9: 05$ & 9.08 & $A G+09803$ & 8.30 & A7 & \\
\hline 456 & $6: 47: 46$ & $15: 57$ & 8.62 & $A G+15689$ & 9.40 & $\mathrm{~A} 5$ & 523 & $6: 55: 02$ & $18: 25$ & 5.71 & $\longrightarrow$ & - & - & \\
\hline 457 & $6: 48: 12$ & $6: 44$ & 7.41 & $\mathrm{BD}+0614021$ & $10.00 \mathrm{P}$ & B... & 524 & $6: 55: 04$ & $5: 17$ & 9.40 & - & - & - & \\
\hline 458 & $6: 48: 16$ & $7: 46$ & 8.71 & $A G+07876$ & 8.60 & B9 & 525 & $6: 55: 05$ & $8: 46$ & 8.07 & $\mathrm{AG}+8874$ & 9.30 & A3 & \\
\hline 459 & $6: 48: 19$ & $1: 06$ & 8.23 & Blend & 8.65 & $\mathrm{~A} 0, \mathrm{~A} 0$ & 526 & $6: 55: 05$ & $1: 55$ & 7.58 & Blend & $8.80 \mathrm{P}$ & $\mathbf{A} 0, \mathbf{A} 0, \mathbf{A} 0$ & \\
\hline 460 & $6: 48: 27$ & 7:04 & 8.40 & - - - & - & - & 527 & $6: 55: 06$ & $14: 42$ & 6.47 & $\mathrm{BD}+141504$ & 10.00 & A0 & \\
\hline 461 & $6: 48: 26$ & $10: 47$ & 8.86 & Blend & 8.35 & $\mathbf{B} 9, \mathbf{A} 2$ & 528 & $6: 55: 07$ & $9: 20$ & 8.87 & $A G+09808$ & 8.80 & A0 & \\
\hline 462 & $6: 48: 27$ & $13: 59$ & 8.01 & $A G+13647$ & 8.10 & KO & 529 & $6: 55: 11$ & $2: 38$ & 9.39 & G $108-42$ & 16.15 & $\mathrm{DC}$ & \\
\hline 463 & $6: 48: 31$ & $1: 15$ & 8.11 & Blend & $7.37 \mathrm{P}$ & $\mathrm{A} 0, \mathrm{A0}, \mathrm{B9}, \mathrm{B8}, \mathrm{A3}, \mathrm{B5}$ & 530 & $6: 55: 16$ & $1: 42$ & 8.47 & HD 51507 & 8.02 & $\mathrm{B3} \mathrm{V}$ & \\
\hline 464 & $6: 48: 35$ & $11: 06$ & 8.23 & - & - & - & 531 & $6: 55: 22$ & $5: 40$ & 9.32 & $\mathrm{BD}+051489$ & 9.70 & $\mathrm{~A}$ & \\
\hline 465 & $6: 48: 36$ & $17: 17$ & 8.45 & —— & - & - & 532 & $6: 55: 44$ & $8: 45$ & 7.94 & Blend & 8.09 & A5, A2 2 & \\
\hline 466 & $6: 48: 42$ & $14: 17$ & 8.64 & - - & - & - & 533 & $6: 56: 01$ & $7: 08$ & 6.47 & $A G+07899$ & 9.70 & A2 & \\
\hline 467 & $6: 48: 49$ & $-0: 26$ & 9.24 & HD 49933 & 5.77 & $\mathrm{~F} 2 \mathrm{~V}$ & 534 & $6: 56: 07$ & $1: 55$ & 7.14 & IID 289425 & $10.00 \mathrm{P}$ & A0 & \\
\hline 468 & $6: 48: 51$ & $14: 29$ & 8.57 & - & $\underline{-}$ & - & 535 & $6: 56: 21$ & $13: 30$ & 9.20 & $\mathrm{BD}+131499$ & 10.40 & $\mathrm{~A} 3$ & \\
\hline 469 & $6: 48: 51$ & $10: 54$ & 8.19 & $A G+10837$ & 9.10 & A2 & 536 & $6: 56: 26$ & $1: 37$ & 9.25 & HD 289489 & $10.00 \mathrm{P}$ & $\mathrm{A} 0$ & \\
\hline 470 & $6: 48: 51$ & $16: 59$ & 7.76 & $A G+16688$ & 8.60 & B9 & 537 & $6: 56: 28$ & $16: 03$ & 7.16 & HD 51889 & $10.00 \mathrm{P}$ & A & \\
\hline 471 & $6: 49: 03$ & $12: 39$ & 8.58 & $A G+12815$ & 9.20 & $A$ & 538 & $6: 56: 35$ & $7: 17$ & 6.94 & HD 51892 & 6.34 & B7III & \\
\hline \multirow[t]{2}{*}{472} & $6: 49: 16$ & $8: 14$ & 8.16 & $A G+08852$ & 8.30 & K0 & 539 & $6: 56: 39$ & $2: 52$ & 8.47 & HD 289402 & $10.00 \mathrm{P}$ & A5 & \\
\hline & & & & & & & 540 & $6: 56: 39$ & $3: 49$ & 9.98 & $A G+03898$ & 8.90 & K5 & \\
\hline
\end{tabular}


TABLE 1 - Continued

\begin{tabular}{|c|c|c|c|c|c|c|c|}
\hline No. & $\alpha_{1950}$ & $\delta_{1850}$ & $m_{1367}$ & Identification & $V$ & Sp. Type & Note \\
\hline 541 & $6: 56: 40$ & $5: 36$ & 8.23 & $\mathrm{AG}+05924$ & 8.60 & $\mathbf{A} 0$ & \\
\hline 542 & $6: 56: 42$ & $10: 05$ & 9.00 & 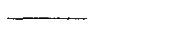 & - & -- & \\
\hline 543 & $6: 57: 12$ & $3: 33$ & 10.24 & $A G+03899$ & 8.40 & A2 & \\
\hline 544 & $6: 57: 18$ & $7: 55$ & 5.83 & -- & $\longrightarrow$ & - & \\
\hline 545 & $6: 57: 27$ & $16: 02$ & 6.03 & HD 51954 & - & A3 & \\
\hline 546 & $6: 57: 30$ & $6: 47$ & 6.40 & $\mathrm{AG}+06851$ & 10.90 & $\mathrm{~A} 2$ & \\
\hline 547 & $6: 57: 31$ & $5: 02$ & 9.67 & - & - & - & \\
\hline 548 & $6: 57: 45$ & $13: 16$ & 9.51 & $\mathrm{BD}+131513$ & 10.40 & $\mathbf{A}$ & \\
\hline 549 & $6: 58: 08$ & $10: 59$ & 8.23 & $\longrightarrow$ & - & 一 & \\
\hline 550 & $6: 58: 22$ & $2: 29$ & 6.25 & Blend & 8.18 & $\mathrm{~A} 0, \mathrm{~A}$ & \\
\hline 551 & $6: 58: 33$ & $13: 11$ & 10.26 & $\mathrm{BD}+131517$ & 10.30 & F2 & \\
\hline 552 & $6: 58: 37$ & $12: 20$ & 9.43 & $\mathrm{BD}+121390$ & 9.70 & $\mathrm{~A} 2$ & \\
\hline 553 & $6: 58: 55$ & $2: 23$ & 6.11 & HD 289462 & $9.50 \mathrm{P}$ & A2 & \\
\hline 554 & $6: 58: 55$ & $10: 45$ & 9.49 & $-\cdots$ & - & - & \\
\hline 555 & $7: 00: 07$ & $6: 10$ & 6.94 & $A G+06857$ & 10.10 & A2 & \\
\hline 556 & $7: 00: 15$ & $12: 18$ & 9.72 & $\mathrm{AG}+12846$ & 8.90 & $\mathrm{~K} 7$ & \\
\hline 557 & $7: 00: 24$ & $7: 40$ & 9.38 & $B D+071568$ & 9.60 & F8 & \\
\hline 558 & $7: 01: 11$ & $9: 22$ & 9.69 & -- & -- & - & \\
\hline 559 & $7: 01: 15$ & $14: 34$ & 8.88 & $\mathrm{BD}+141543$ & 10.30 & F8 & \\
\hline 560 & $7: 01: 31$ & $15: 27$ & 9.39 & Blend & 6.99 & $\mathrm{~A} 2, \mathrm{~B} 9$ & \\
\hline 561 & $7: 01: 38$ & $3: 45$ & 8.80 & $\mathrm{AG}+03923$ & 9.00 & $\mathrm{~B} 8$ & \\
\hline 562 & $7: 01: 58$ & $8: 54$ & 9.03 & $\ldots$ & - & - & \\
\hline 563 & $7: 02: 29$ & $10: 02$ & 9.38 & & - & 一 & \\
\hline 564 & $7: 02: 44$ & $5: 10$ & 8.22 & $\longrightarrow$ & - & 一 & \\
\hline 565 & $7: 02: 49$ & $6: 41$ & 7.68 & $\mathrm{AG}+06864$ & 10.50 & F5 & \\
\hline 566 & $7: 02: 54$ & $7: 18$ & 9.10 & Blend & 8.46 & $\mathrm{B9}, \mathrm{B8}$ & \\
\hline 567 & $7: 02: 55$ & $15: 04$ & 6.53 & HD 53449 & -- & A & \\
\hline 568 & $7: 02: 59$ & $1: 56$ & 7.51 & $A G+01837$ & 8.90 & B9 & \\
\hline 569 & $7: 03: 11$ & $4: 35$ & 9.51 & $\mathrm{AG}+04907$ & 9.70 & F2 & \\
\hline 570 & $7: 03: 14$ & $11: 51$ & 8.41 & $\mathrm{AG}+11797$ & 9.60 & K0 & \\
\hline 571 & $7: 03: 17$ & $4: 16$ & 9.30 & - - & - & - & \\
\hline $\mathbf{5 7 2}$ & $7: 03: 25$ & $6: 28$ & 6.09 & G $108-54$ & 16.20P & - & $*$ \\
\hline 573 & $7: 03: 39$ & $5: 55$ & 9.35 & $\mathrm{BD}+061512$ & 9.50 & AO & \\
\hline 574 & $7: 03: 40$ & $2: 41$ & 8.09 & Blend & $8.75 \mathrm{P}$ & $\mathrm{G} 5, \mathrm{~K} 0$ & \\
\hline 575 & $7: 04: 18$ & $14: 01$ & 8.24 & Blend & - & $\mathrm{A}, \mathrm{F5}$ & \\
\hline 576 & $7: 04: 32$ & $7: 22$ & 9.43 & $\mathrm{BD}+071602$ & 9.70 & F8 & \\
\hline 577 & $7: 04: 45$ & $6: 47$ & 8.75 & $-\cdots$ & - & - & \\
\hline 578 & $7: 04: 47$ & $9: 16$ & 8.56 & $A G+09834$ & 9.10 & AO & \\
\hline 579 & $7: 05: 31$ & $5: 32$ & 5.67 & $\mathrm{AG}+05951$ & 10.20 & A5 & \\
\hline 580 & $7: 05: 56$ & $7: 02$ & 9.63 & $A G+07932$ & 10.60 & $\mathbf{A} 0$ & \\
\hline 581 & $7: 06: 21$ & $8: 34$ & 8.19 & $-\cdots$ & $\ldots$ & - & \\
\hline 582 & $7: 06: 21$ & $7: 46$ & 8.79 & Blend & 9.11 & $\mathrm{~A} 0, \mathrm{~B} 8$ & \\
\hline 583 & $7: 06: 29$ & $3: 12$ & 7.84 & - - & - & - & \\
\hline 584 & $7: 06: 42$ & $6: 35$ & 9.21 & Blend & 7.74 & $\mathrm{~B} 9, \mathrm{~A} 2$ & \\
\hline 585 & $7: 06: 48$ & $3: 54$ & 9.50 & $\mathrm{AG}+04922$ & 9.80 & A0 & \\
\hline 586 & $7: 07: 33$ & $8: 29$ & 8.82 & $\mathrm{AG}+08924$ & 10.00 & A2 & \\
\hline 587 & $7: 08: 09$ & $3: 56$ & 9.49 & $A G+04925$ & 9.80 & A2 & \\
\hline 588 & $7: 08: 23$ & $6: 45$ & 8.47 & $\mathbf{A G}+06 \mathbf{8 8 5}$ & 9.10 & A0 & \\
\hline 589 & $7: 08: 26$ & $8: 08$ & 5.71 & $\overline{-}$ & $\longrightarrow$ & - & \\
\hline 590 & $7: 08: 34$ & $9: 29$ & 9.09 & SKY 12927 & 7.50 & B9 & \\
\hline 591 & $7: 08: 42$ & $10: 04$ & 8.23 & $-\ldots$ & - & - & \\
\hline 592 & $7: 08: 45$ & $6: 58$ & 8.87 & $A G+06888$ & 8.20 & FoV & \\
\hline 593 & $7: 09: 10$ & $14: 40$ & 7.19 & $\mathbf{A G}+14765$ & 8.50 & AO & \\
\hline 594 & $7: 09: 41$ & $7: 43$ & 7.33 & Blend & 9.00 & $\mathrm{~A} 0, \mathbf{B} 8$ & \\
\hline 595 & $7: 09: 52$ & $9: 30$ & 8.72 & $A G+09851$ & 10.40 & A2 & \\
\hline 596 & $7: 10: 09$ & $13: 13$ & 8.82 & $A G+13701$ & 9.20 & F5 & \\
\hline 597 & $7: 10: 21$ & $6: 18$ & 7.51 & $\overline{\mathrm{nO}}$ & $\longrightarrow$ & - & \\
\hline 598 & $7: 12: 37$ & $7: 55$ & 8.00 & $\mathrm{BD}+081711$ & $9.30 \mathrm{P}$ & 一 & \\
\hline 599 & $7: 12: 52$ & $8: 44$ & 7.16 & $\mathbf{A G}+08941$ & 10.10 & A 0 & \\
\hline 600 & $7: 13: 01$ & $11: 44$ & 7.61 & $\ldots$ & $-\ldots$ & - & \\
\hline 601 & $7: 14: 14$ & $8: 17$ & 8.48 & $\mathrm{AG}+08946$ & 8.40 & Ko & \\
\hline 602 & $7: 15: 21$ & $11: 53$ & 9.02 & -— & - & - & \\
\hline
\end{tabular}

which stars to include in the blends, we ignored stars which were more than 2.5 mag fainter than the brightest star in the group (in the visible), and we ignored stars more than a spectral class later than the brightest star. In many cases, there was not an object in the circle of confusion which was bright enough to plausibly account for the uv object. In that case the identification is left blank. The sixth column of Table 1 gives the magnitude of the identified star from the SIMBAD data base. Most of these are $V$ magnitudes but when only photographic magnitudes are present these have been listed followed by a $P$. In cases of blends, the combined visual magnitude of all the stars which might reasonably be expected to contribute to the ultraviolet flux is given. The spectral types from the data base are listed in the seventh column. For blends, spectral types for the stars which were included in the blend are listed in order of brightness.

In cases with only faint or late-type stars in the circle of confusion the source of the uv flux is uncertain and some judgment was employed to decide whether to include them as identifications. In those cases where a faint or a late star is given as the identification, it should be borne in mind that the actual source of the uv flux could easily be another star not contained in the SIMBAD data base. However, there are also many known cases of cool stars which are bright in the ultraviolet due to a hot companion which is faint (and perhaps not previously detected) in the visible. Further observations are needed to clarify the situation for many of the objects in Table 1.

\section{DISCUSSION}

There are 11 objects in Table 1 which are associated with the star clusters NGC 2169 (object 47), NGC 2244 (object 242, 243, 255,260 , and 267), and NGC 2264 (object 337, 340, 362, 364, and 366). These clusters are too crowded to allow the resolution of individual stars and appear as one or more clumps on our images. In Figure 3 we show contour plots of the region of each cluster. The contours from two different exposures are provided to show details in both the fainter and the brighter regions. Some effects of saturation are apparent in the long-exposure images. Although aperture photometry is inaccurate for such objects, we give magnitudes in Table 1 for the various clumps as a rough guide to the distribution of flux. In the footnotes to the table we also give the integrated magnitudes NGC 2169 and NGC 2244. These were obtained with a polygonal aperture which covered the area of the cluster. In the

Notes ON INDIVIDUAL OBIECTS.-

47: NGC 2169, individual stars not resolved. The integrated uv magnitude of the cluster is $m_{1367}=4.82$.

$242,243,255,260,267: \mathrm{NGC} 2244$, individual stars not resolved. The integrated uv magnitude of the cluster is $m_{1367}=4.62$.

337: The $V$ magnitude and spectral type refer to the star NGC 2264-7 which dominates this object.

340: The $V$ magnitude and spectral type refer to the star NGC 2264-24 which dominates this object.

362 and 366: Unresolved clumps of stars in NGC 2264.

364: This group is dominated by 15 Mon (HD 47839, S Mon) to which the $V$ magnitude and spectral type refer.

377: Two brighter stars within the circle of confusion are late types: K0 with $P=8.5$ and G5 with $P=9.1$. We assume that the uv fux is from the $B$ star.

450: Five faint $A$ and $B$ stars in the circle of confusion.

467: There are several B and A stars in the circle of confusion which are more than 4 mag fainter than the F2 V star.

505: Eight A and B stars with $P \sim 10$ in the circle of confusion.

572: This star is apparently a white dwarf based on its proper motion. 


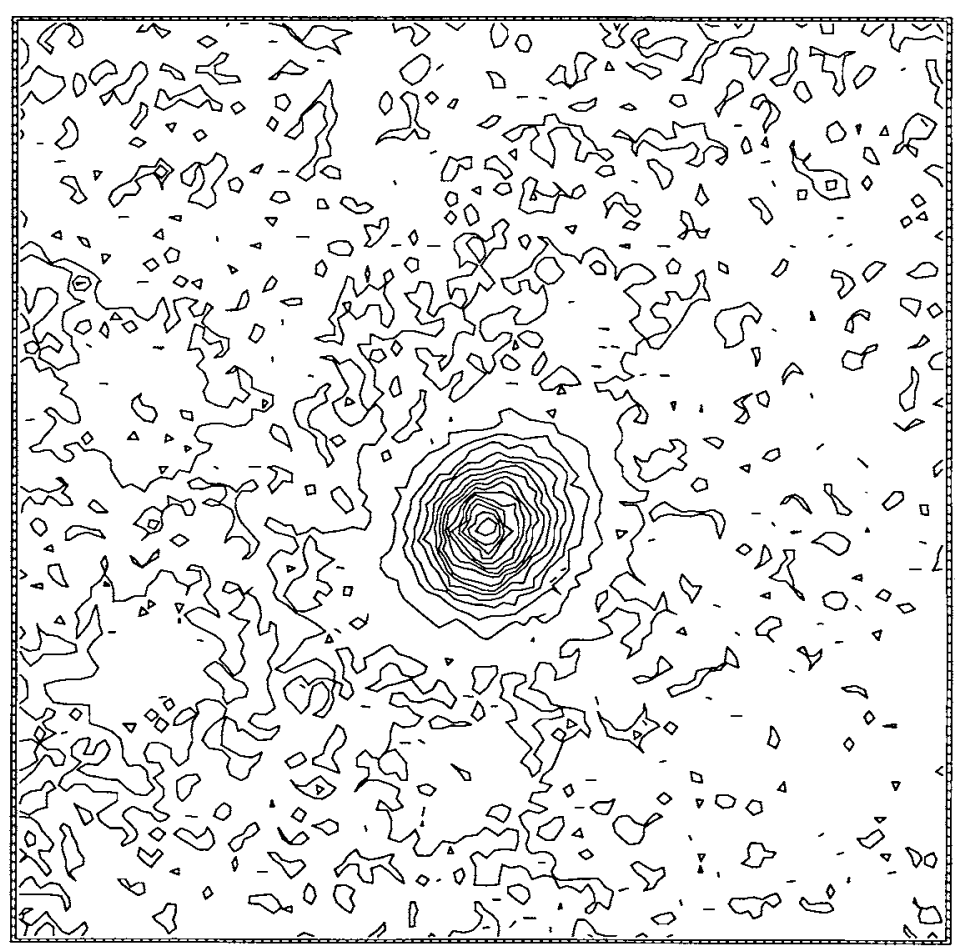

FIG. $3 a$

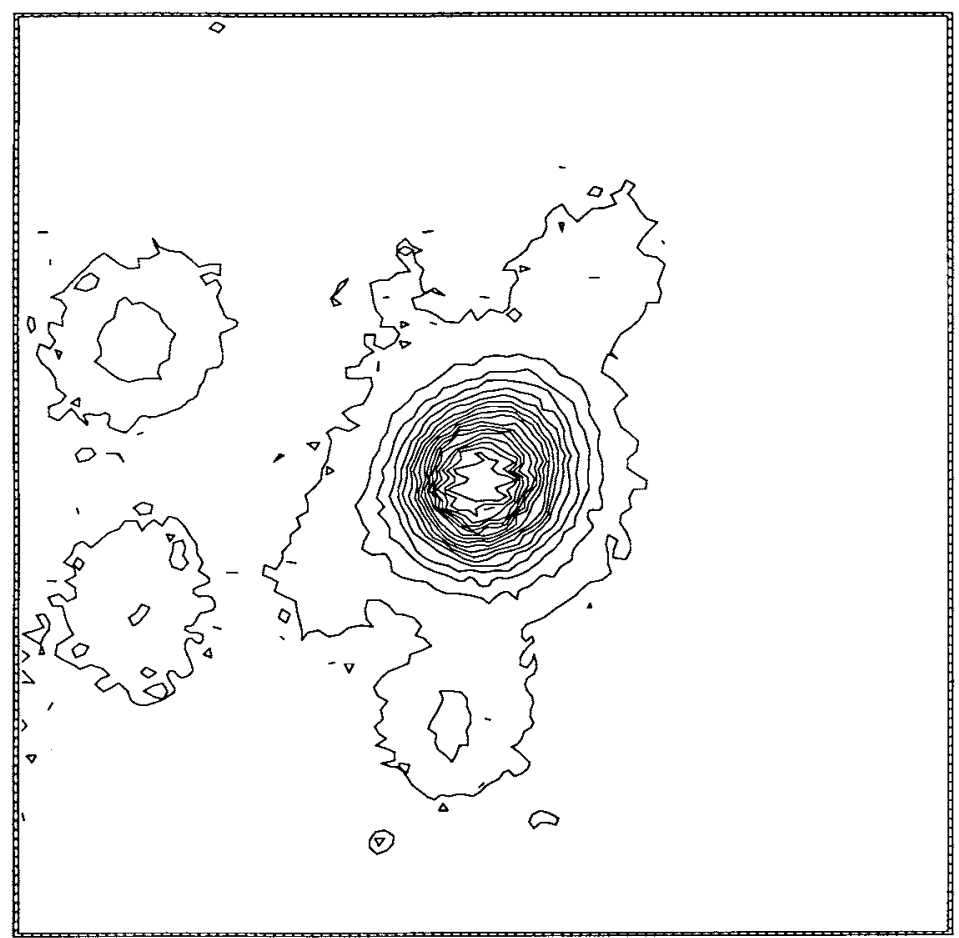

Fig. $3 b$

Fig. 3.--Isodensity plots for the three star clusters. (a) NGC 2169 from the $19: 5$ and $99: 6$ exposures, (b) NGC 2244 from the 19.5 and 99.6 exposures, (c) NGC 2264 from the 4.4 and 99:6 exposures. In panel $a$ an area an arcminute on a side is shown while panels $b$ and $c$ show areas 1:5 on a side. 


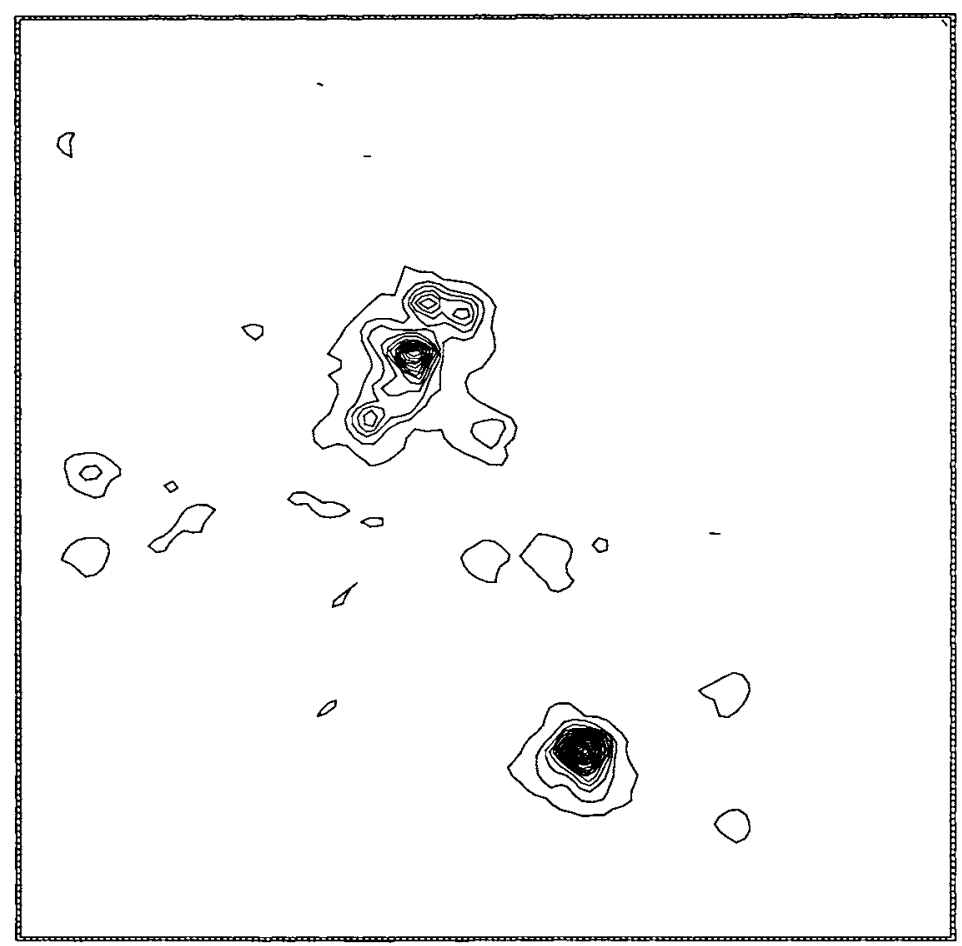

FIG. $3 c$

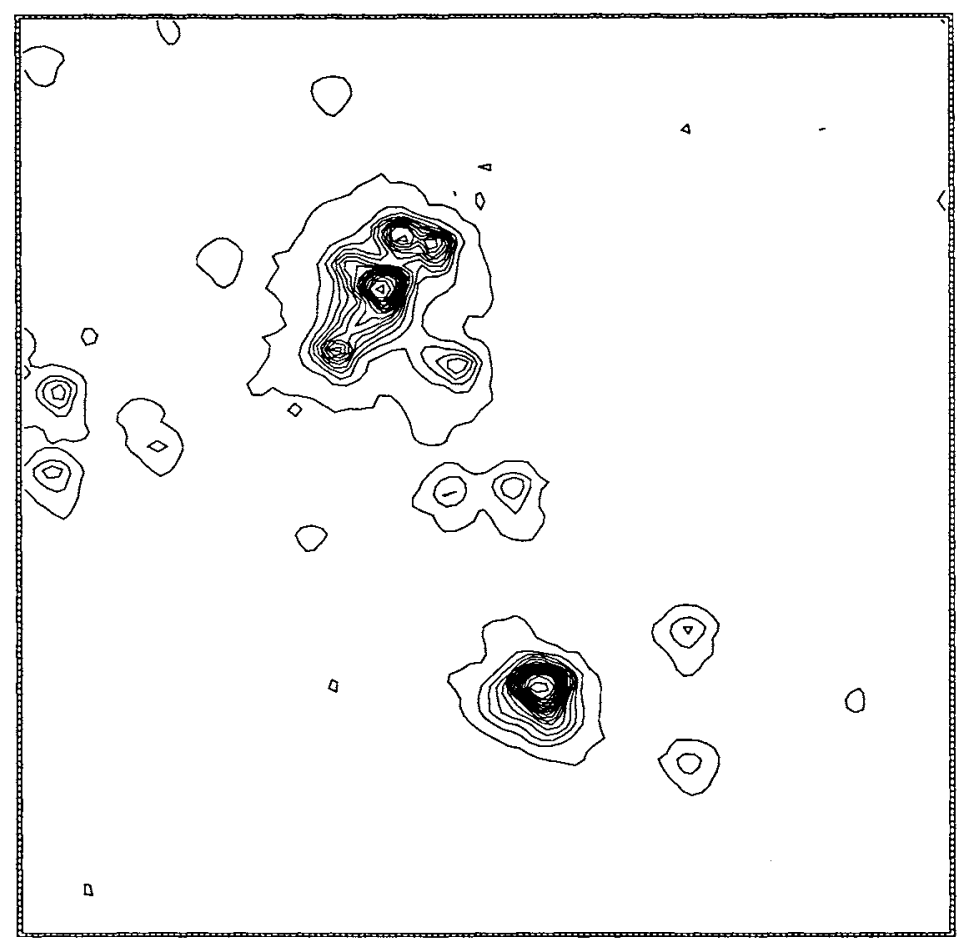

FiG. $3 d$

case of NGC 2264, 15 Mon (S Mon, HD 47839) dominates the ultraviolet flux. As noted above, it is too saturated to allow a reliable estimate of its magnitude from the electrographic images. We have thus obtained the integrated magnitude of the remainder of the cluster area and combined it with the magnitude for 15 Mon in Table 1 to obtain an integrated magnitude of $m_{1367}=1.12$ for $\mathrm{NGC} 2264$.
An examination of Table 1 shows that $58 \%$ of the objects have been identified with visible stars. An additional $25 \%$ are labeled as blends. In the following discussion we will be mainly concerned with a subsample for which we were able to obtain optical data from SIMBAD. We have omitted all the objects identified as blends or with star clusters. Only stars with $V$ magnitudes and those for which a spectral class and subclass 


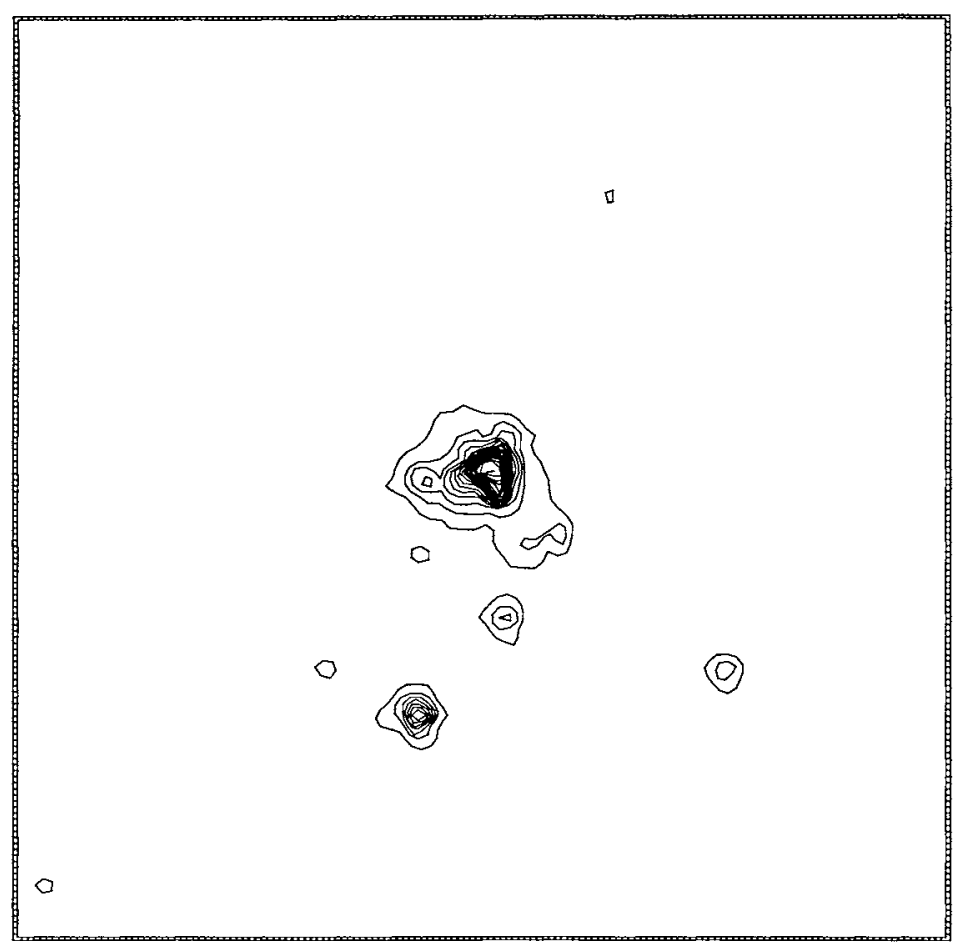

FIG. $3 e$

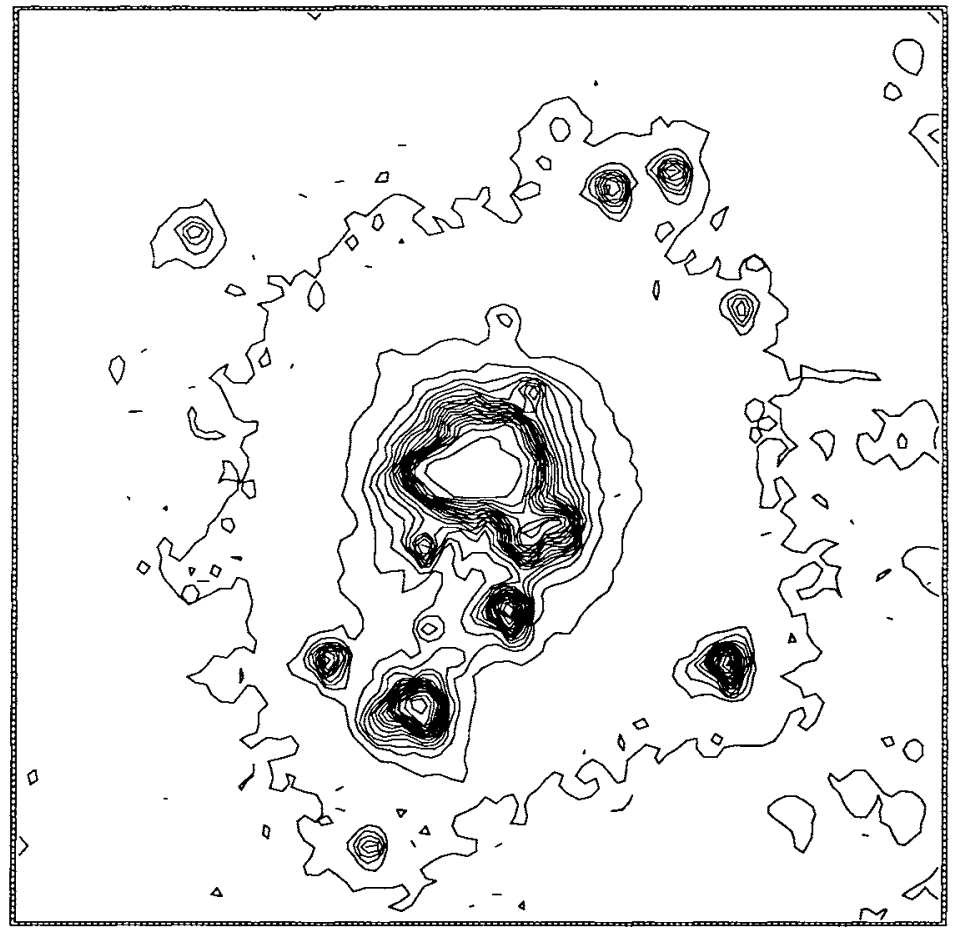

Fig. $3 f$

are available were included. The resulting selected sample contains 300 stars.

In Figure $4 a$ we show a histogram of the frequency distribution of the stars in ultraviolet magnitude for both the full sample and the selected sample. It can be seen that the distributions of the two samples are nearly identical. Thus our comments regarding the selected sample should apply to the full sample as well. In Figure $4 b$ we show the cumulative magnitude as a function of limiting magnitude for the full sample.

In previous studies (Carruthers \& Page 1984c) it was found that in fields near the Galactic plane the frequency distribution increased rapidly toward fainter stars to within a magnitude or 


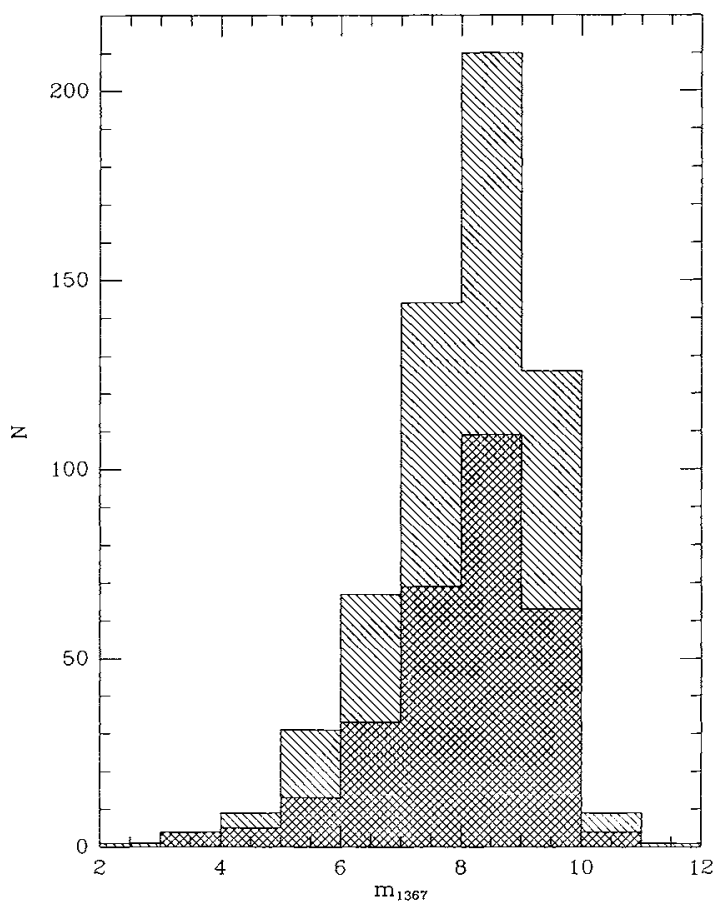

Fig. $4 a$

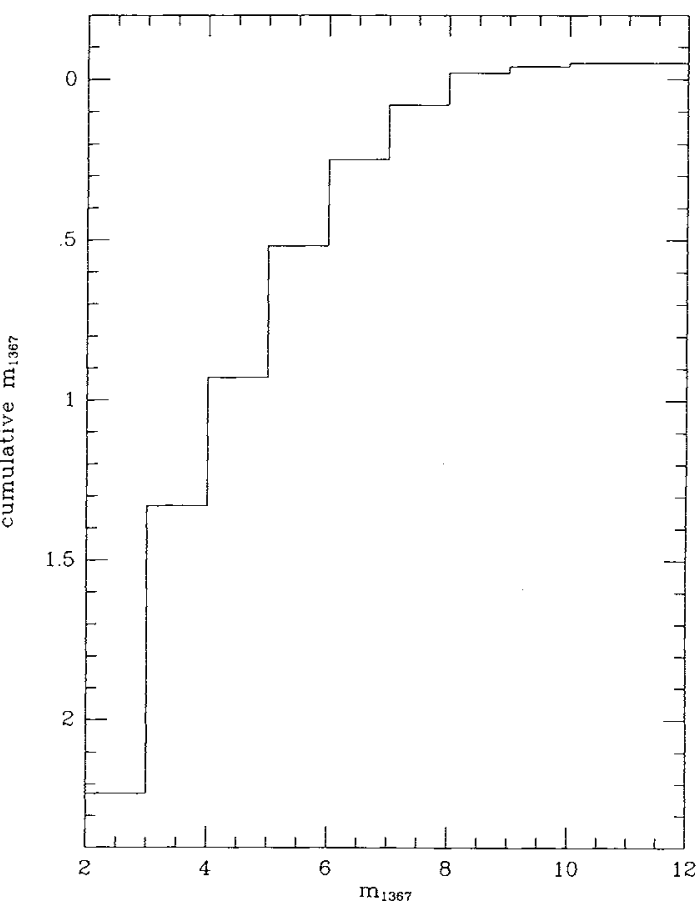

FiG. $4 b$

Fig. 4. - (a) Number of objects from Table 1 in 1 mag intervals vs. ultraviolet magnitude, $m_{1367}$. The darker shaded region indicates those objects included in the selected sample. $(b)$ The cumulative magnitude of all the objects in Table 1 as function of limiting magnitude.

so of the plate limit. Consequently, the faint stars contributed significantly to the ultraviolet flux. On the other hand, in highlatitude fields, the distribution was flatter and the brightest stars contributed most of the ultraviolet flux. The distributions shown in Figure 4 are in accord with these previous findings since Monoceros is on the Galactic plane.

In Figures $5 a$ and $5 b$ we plot the distributions of the selected sample in terms of spectral type and color index, $\left(m_{1367}-V\right)$. As was found in the previous studies, the largest number of the stars in the sample are classed as A0. A difference from the corresponding plot shown by Carruthers \& Page (1984c) is the inclusion here of a significant number of later spectral types. About $20 \%$ of the selected sample are of type F0 or later. While

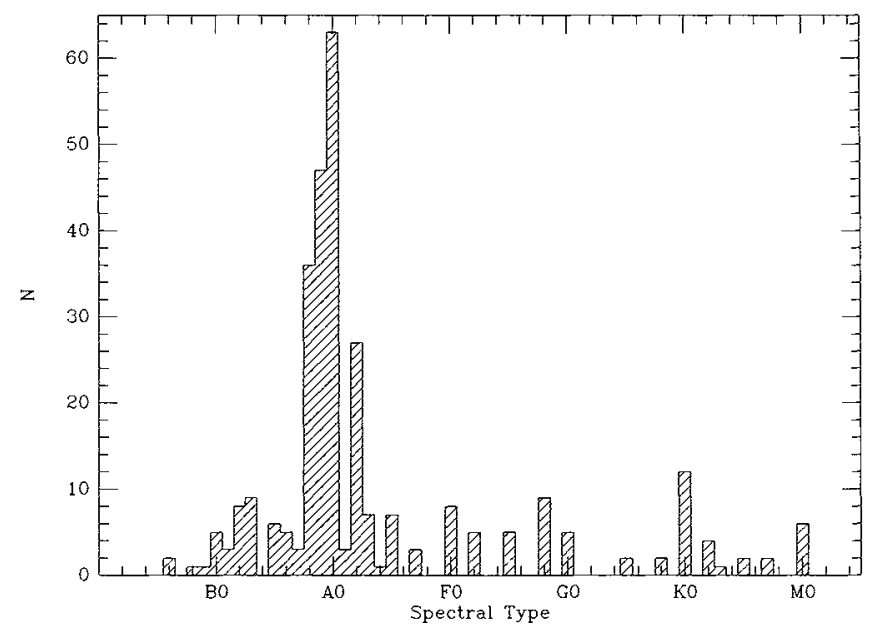

FIG. $5 a$ some of these stars are certainly misidentifications, there are no doubt many examples among them of cool stars with faint, hot companions. On the other hand, those ultraviolet objects which have been associated with late-type stars through misidentification as well as the stars lacking a SIMBAD identification are likely to be objects which are faint in the visible but bright in the ultraviolet. Efforts should be made to identify them in the visible as they promise to be objects of special interest.

In Figure 6 we have plotted $\left(m_{1367}-V\right)$ against the visual magnitude for stars with spectral classes of $\mathrm{B} 8, \mathrm{~B} 9$, and $\mathrm{A} 0$. The limiting magnitude of our observations varies across the field but from an examination of Figure $4 a$ we estimate that it is

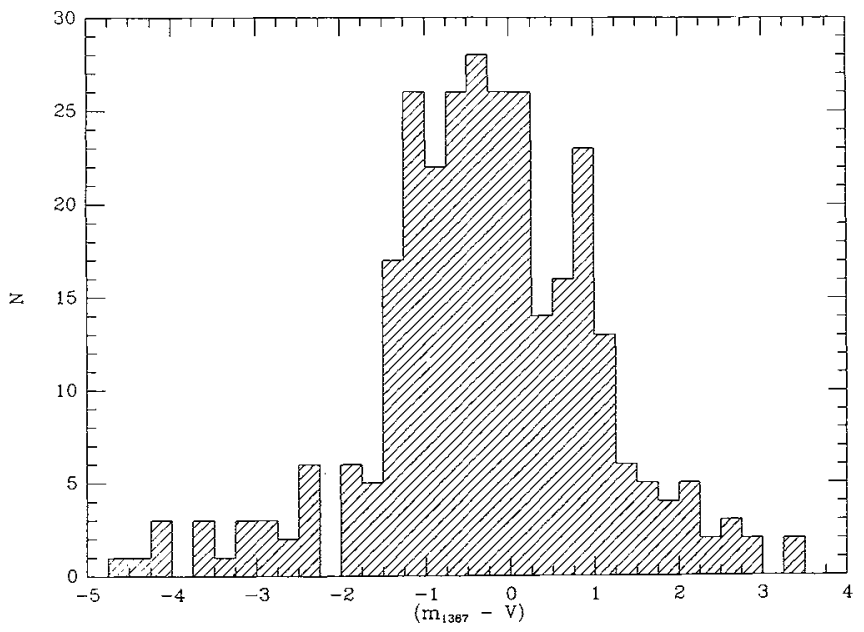

FIG. $5 b$

FIG. 5.-The frequency distributions of the stars in our selected sample as a function of spectral type and the $\left(m_{1367}-V\right)$ color index 


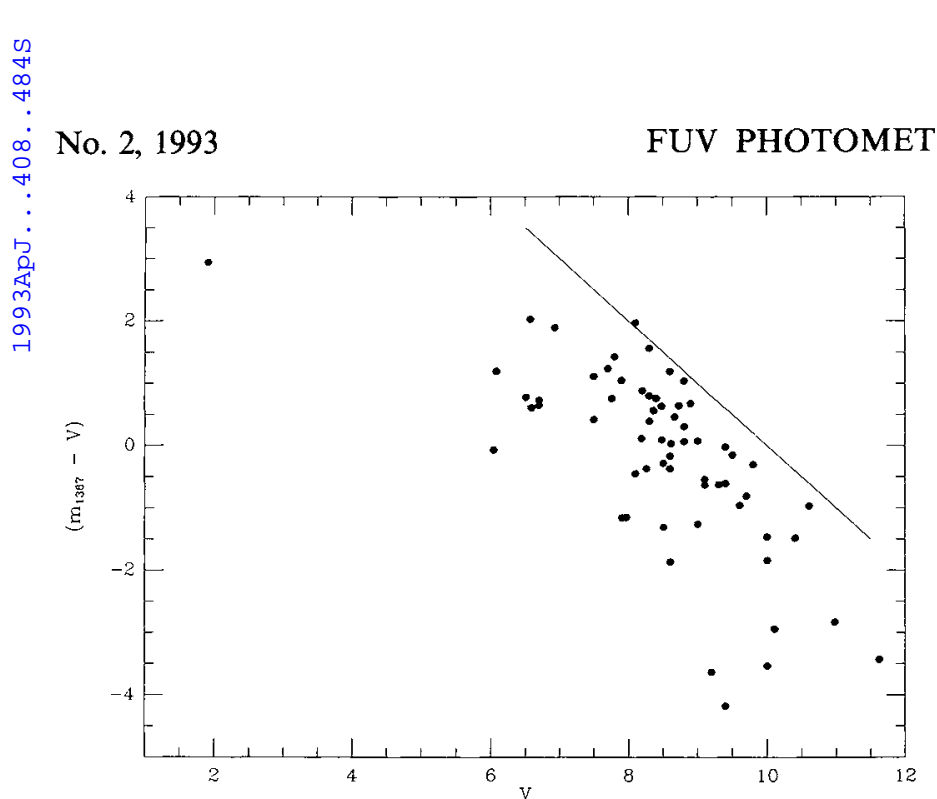

FIG. 6.-A plot of the $\left(m_{1367}-V\right)$ color index against the $V$ magnitudes for stars in the selected sample with spectral types of $\mathrm{B} 8, \mathrm{~B} 9$, and $\mathrm{A} 0$. The diagonal line in the upper right corner of the plot represents a limiting magnitude in the ultraviolet of 10 .

generally near $m_{1367}=10$. The diagonal line in the upper right corner of Figure 6 represents this limit. The bulk of the stars in this diagram are located in a large group between $V=6$ and $V=10$ and with $\left(m_{1367}-V\right)$ between -1.5 and +1.5 . Most of these stars are probably main-sequence stars at distances up to a kiloparsec. Since the visual absorption in this field ranges from 0.3 to $1.9 \mathrm{mag} \mathrm{kpc}^{-1}$ (Sharov 1964), the color excess in $\left(m_{1367}-V\right)$ can range up to $3.6 \mathrm{mag}$ (using 3 for the ratio of absorption at $1396 \AA$ to that in the visible following Mathis 1990). Variations in the absorption across the field are thus adequate to account for the vertical scatter of this group of stars although the range in spectral types and errors in the spectral types must also contribute.

There is a group of six stars in the lower right corner of Figure 6 (objects 294, 400,447, 455, 527, 599). These objects have color indices ranging from -2.8 to -4.2 and visual magnitudes in the range from 9 to 12 . Neglecting reddening this implies temperatures in the range of $15,000 \mathrm{~K}$ to $30,000 \mathrm{~K}$. Hot main-sequence stars with $V$ near 10 would have distances in excess of $1.5 \mathrm{kpc}$. Thus they should be substantially reddened.
The other alternative is to assume these stars are relatively nearby, low-luminosity stars. We suggest that the six stars in this group are white dwarfs. Only one of them, star 455 in Table 1, has an MK spectral classification. The spectral type, $\mathrm{A} 0 \mathrm{~V}$, is inconsistent with a white dwarf and should be verified.

A number of objects from Table 1 would fall in the region of the low-luminosity stars but are not plotted in Figure 6 because their spectral types fall outside of the range from B8 to A0. Objects $533,546,555,565$, and 579 are only slightly outside of that range and probably belong with the six stars discussed in the previous paragraph. Objects $68,285,290$, and 347 fall between the low-luminosity group and the other stars in Figure 6; their status is thus ambiguous.

There are three additional objects in Table 1 which did not meet the spectral type criterion for inclusion in Figure 6 but which appear similar to the six low-luminosity objects discussed in the previous paragraphs. Object 84 is identified as a planetary nebula, while objects 529 and 572 are apparent white dwarfs. Object 84 is a magnitude fainter than any of the stars in Figure 6 but has a color, $\left(m_{1367}-V\right)=-3.23$ which matches the low-luminosity group. This is compatible with our contention that these stars are hot, low-luminosity objects. On the other hand, objects 529 and 572 are both considerably fainter in the visible than the stars in the low-luminosity group in spite of relatively bright ultraviolet magnitudes. Evidently they are much hotter stars.

Further analysis should include more detailed consideration of those objects in our list for which more accurate spectral types, visual magnitudes and $E(B-V)$ values are available. It should also include acquisition of such data for objects in our list which appear peculiar.

We thank Harry M. Heckathorn, Brian C. Dohne, and Darwin E. Reiswig for assistance in the data analysis and useful discussions. We also acknowledge contributions by Chet B. Opal (deceased) to the experiment planning and development and by David King and Harry Merchant (deceased) in preflight technical support. The $I U E$ Regional Data Analysis Facility at Goddard Space Flight Center and the SIMBAD data base, operated by CDS, Strasbourg, France, were used in this research.
Carruthers, G. R. 1973, Appl. Optics, 12, 2501

Carruthers, G. R., \& Opal, C. B. 1977, ApJ, 212, L27

Carruthers G. R., \& Page, T. 1983, ApJS, 53, 623

- 1984a, ApJS, 54, 271

1984 b, ApJS, 55,101

1984c, PASP, 96, 447

\section{REFERENCES}

Egret, D., Wenger, M., \& Dubois, P. 1991, in Databases and On-line Data in Astronomy, ed. M. A. Albrecht \& D. Egret (Dordrecht: Kluwer), 79 Mathis, J. S. 1990, ARA\&A, 28, 37

Sharov, A. S. 1964, Soviet Astron. 7, 689 (AZh, 40, 900, 1962)

Stetson, P. B. 1987, PASP, 99, 191

Tody, D. 1986, Proc. SPIE, 627, 733 
PLATE 8

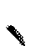

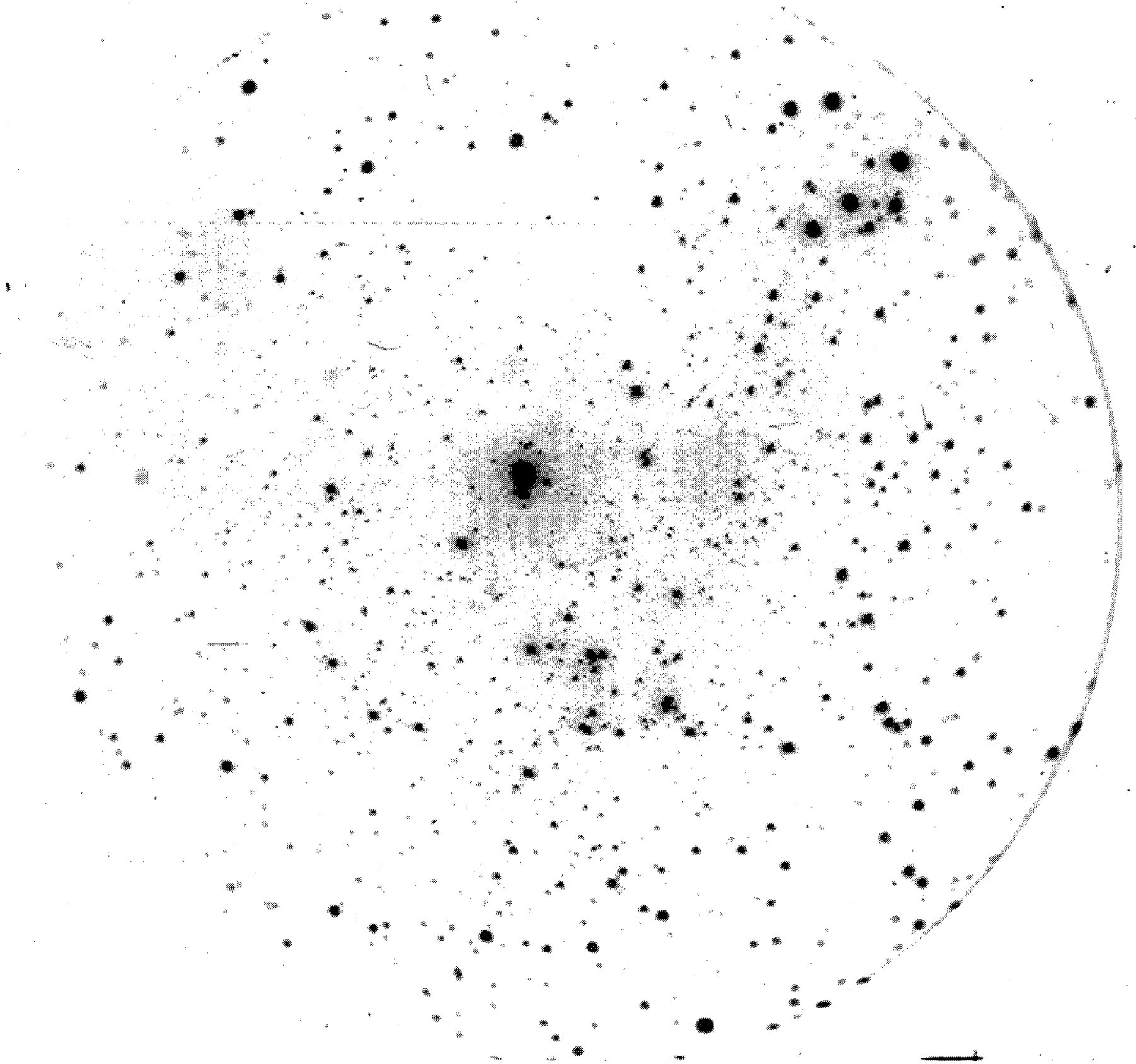

FIG. 2.-A print of the $99^{\mathrm{s}} .6$ exposure. The field center is at $\alpha_{1950}=6^{\mathrm{h}} 35^{\mathrm{m}}, \delta_{1950}=9^{\circ} 10^{\prime}$. The bright clump near the field center is the bright $\mathrm{O} 7 \mathrm{Ve}$ star $15 \mathrm{Mon}$ and other stars in the cluster NGC 2264. North is at the top, and east to the left.

SChIMdT \& CARRUThers (see 408, 484) 